ESAIM: COCV 27 (2021) 69

https://doi.org/10.1051/cocv/2021066
ESAIM: Control, Optimisation and Calculus of Variations

www.esaim-cocv.org

\title{
OPEN-LOOP AND CLOSED-LOOP SOLVABILITIES FOR STOCHASTIC LINEAR QUADRATIC OPTIMAL CONTROL PROBLEMS OF MARKOVIAN REGIME SWITCHING SYSTEM*
}

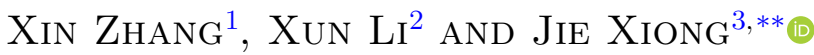

\begin{abstract}
This paper investigates the stochastic linear quadratic (LQ, for short) optimal control problem of Markovian regime switching system. The representation of the cost functional for the stochastic LQ optimal control problem of Markovian regime switching system is derived by the technique of Itô's formula with jumps. For the stochastic LQ optimal control problem of Markovian regime switching system, we establish the equivalence between the open-loop (closed-loop, resp.) solvability and the existence of an adapted solution to the corresponding forward-backward stochastic differential equation with constraint. (i.e., the existence of a regular solution to Riccati equations). Also, we analyze the interrelationship between the strongly regular solvability of Riccati equations and the uniform convexity of the cost functional. Finally, we present an example which is open-loop solvable but not closed-loop solvable.
\end{abstract}

Mathematics Subject Classification. 49N10, 93E20.

Received March 22, 2020. Accepted June 12, 2021.

\section{INTRODUCTION}

Linear-quadratic (LQ) optimal control problem plays an important role in control theory. It is a classical yet fundamental problem in the fields of control theory. In the past few decades, both the deterministic and stochastic linear quadratic (LQ) control problems have been widely studied. Stochastic LQ optimal control problem was first carried out by Kushner [12] via dynamic programming method. Later, Wonham [27] studied the generalized version of the matrix Riccati equation arose in the problems of stochastic control and filtering. Using functional analysis techniques, Bismut [1] proved the existence of the Riccati equation and derived the existence of the optimal control in a random feedback form for stochastic LQ optimal control with random coefficients. Tang [24] discussed the existence and uniqueness of the associated stochastic Riccati equation for

${ }^{*}$ The first author is supported by National Natural Science Foundation of China (grant no. 11771079) and Fundamental Research Funds for the Central Universities (grant no. 2242021R41082), the second author is supported by RGC Grants (grant nos. 15209614, 15213218 and 15215319) and partially from CAS AMSS-PolyU Joint Laboratory of Applied Mathematics, and the third author is supported by Southern University of Science and Technology Start up fund Y01286120 and National Natural Science Foundation of China (grant nos. 61873325, 11831010).

Keywords and phrases: Linear quadratic optimal control, markovian regime switching, riccati equations, open-loop solvability, closed-loop solvability.

1 School of Mathematics, Southeast University, Nanjing, Jiangsu Province 211189, PR China.

2 Department of Applied Mathematics, The Hong Kong Polytechnic University, Hong Kong, PR China.

3 Department of Mathematics, Southern University of Science and Technology, Shenzhen, Guangdong 518055, PR China.

** Corresponding author: xiongj@sustech.edu.cn

(C) The authors. Published by EDP Sciences, SMAI 2021

This is an Open Access article distributed under the terms of the Creative Commons Attribution License (https://creativecommons.org/licenses/by/4.0), which permits unrestricted use, distribution, and reproduction in any medium, provided the original work is properly cited. 
general stochastic LQ optimal control problems with random coefficients and state control dependent noise via the method of stochastic flow, which can be applied to Bismut and Peng's long-standing open problems. Moreover, Tang provided a rigorous derivation of the interrelationship between the Riccati equation and the stochastic Hamilton system as two different but equivalent tools for the stochastic LQ problem. For more details on the progress of the stochastic Riccati equation, interested readers may refer to [8-11, 21, 25].

Under some mild conditions on the weighting coefficients in the cost functional, such as positive definite quadratic weighting control martix, and so on, the stochastic LQ optimal control problems can be solved elegantly via the Riccati equation approach, see Chapter 6 of [30]. Chen et al. [2] was the first to start the pioneering work of stochastic LQ optimal control problems with indefinite quadratic weighting control matrix, which turns out to be useful in solving the continuous-time mean-variance portfolio selection problems. Since then, there has been an increasing interest in the so-called indefinite stochastic LQ optimal control, see, for example, Chen and Yong [3], Li and Zhou [14], Li et al. [15, 16], and so on.

Another extension to stochastic LQ optimal control problems involves random jumps in the state systems, such as Poisson jumps or the regime switching jumps. Wu and Wang [28] considered the stochastic LQ optimal control problems with Poisson jumps and derived the existence and uniqueness of the deterministic Riccati equation. Using the technique of completing squares, Hu and Oksendal [4] discussed the stochastic LQ optimal control prblmlem with Poisson jumps and partial information. Existence and uniqueness of the stochastic Riccati equation with jumps and connections between the stochastic Riccati equation with jumps and the associated Hamilton systems of stochastic LQ optimal control problem were also presented. Yu [31] investigated a kind of infinite horizon backward stochastic LQ optimal control problems and differential game problems under the jump-diffusion model state system. Li et al. [13] solved the indefinite stochastic LQ optimal control problem with Poisson jumps.

The stochastic control problems involving regime switching jumps are of interest and of practical importance in various fields such as science, engineering, financial management and economics. The past few years have witnessed a dramatically increasing interest in financial market models whose key parameters, such as interest rate, stocks return and volatility, are modulated by Markov processes. In particular, one could face two market regimes in financial markets, one of which stands for a bull market with price increase, while the other for a bear market with price drop. One such formulation is the so-called regime switching model, where the market parameters depend on market modes that switch among a finite number of regimes. More recently, the regime switching models and related topics have been extensively studied in the areas of stochastic controls as well as their financial applications, see, for examples, [14-16, 19, 29, 35-39]. Ji and Chizeck [6, 7] formulated a class of continuous-time LQ optimal controls with Markovian jumps. Zhang and Yin [34] developed hybrid controls of a class of LQ systems modulated by a finite-state Markov chain. Li and Zhou [14], Li et al. [15, 16] introduced indefinite stochastic LQ optimal controls with regime switching jumps. Liu et al. [17] considered near-optimal controls of regime switching LQ problems with indefinite control weight costs.

Recently, Sun and Yong [22] investigated the two-person zero-sum stochastic LQ differential games. It was shown in Sun and Yong [22] that the open-loop solvability is equivalent to the existence of an adapted solution to a forward-backward stochastic differential equation (FBSDE, for short) with constraint and closed-loop solvability is equivalent to the existence of a regular solution to the Riccati equation. As a continuation work of Sun and Yong [22], Sun et al. [20] considered the open-loop and closed-loop solvabilities for stochastic LQ optimal control problems. Moreover, the equivalence between the strongly regular solvability of the Riccati equation and the uniform convexity of the cost functional was established. This naturally calls for us to study the open-loop and closed-loop solvabilities within the framework of regime switching jumps. Moreover, if there is only one regime jump state in our model and then it can be reduced to that model of Sun et al. [20]. The aim of this paper is to further develop the results in Sun et al. [20] to the case of stochastic LQ optimal control problems with regime switching jumps. Although the only difference between our model and their one is the regime switching jumps, it is not easy for one to obtain the similar results. The first difficulty we encounter is the uniformly convexity characterization of the cost functional $J(t, x, i ; u(\cdot))$. In Sun et al. [20], however, it is hard to understand the relationships of the uniformly convexity among $J^{0}(t, x ; u(\cdot)), J^{0}(t, 0 ; u(\cdot))$ and $J(t, x ; u(\cdot))$, because they just briefly mentioned "uniformly convexity" and did not clearly prsent readers its fundamental 
definition. Thus, one cannot directly deal with the similar control works including the regime switching case. Therefore, in our work, we definitely and strictly introduce the definition of "uniformly convexity" from Zalinescu $[32,33]$. Then, using this definition, we prove that the uniformly convexity of the cost functional $J(t, x, i ; u(\cdot))$ is equivalent to the positive definiteness of the operator $M_{2}(t, i)$ (see (3.2) or (3.8)). From this equivalence, we can further obtain the relationships of the uniformly convexity among $J^{0}(t, x, i ; u(\cdot)), J^{0}(t, 0, i ; u(\cdot))$ and $J(t, x ; u(\cdot))$. The second difficulty in our model is to prove the equivalence between the closed-loop solvability and the existence of regular solution to Riccati equation. In the model without regime switching jumps, this equivalence was studied in Sun and Yong [22] and Sun et al. [20]. In order to prove the equivalence, Sun and Yong [22] constructed two matrix valued stochastic processes $\mathbb{X}(\cdot)$ and $\mathbb{Y}(\cdot)$ (see Eqs. (5.16) and (5.17) in [22]) and set $P(s):=\mathbb{Y}(s) \mathbb{X}(s)^{-1}$. Then the authors prove that $P(s)$ satisfies the Riccati equation. In our case, note that solution $P(s, i)$ of Riccati equation (5.2) depends on state $\alpha(s)=i$. However, $\mathbb{Y}(s) \mathbb{X}(s)^{-1}$ may not equal to $P(s, \alpha(s))$ even though the evolution of the matrix valued processes $\mathbb{Y}(s)$ and $\mathbb{X}(s)$ depend on the Markov chain $\alpha$. Thus, if we adopt the technique in Sun and Yong [22], we may not parallelly derive $P(s, \alpha(s))$. Therefore, the method adopted in [22] no longer works in our model. Wang [26] provided a new method to avoid the procedures of defining $P(s):=\mathbb{Y}(s) \mathbb{X}(s)^{-1}$ for the model without regime switching. The method adopted by [26] can also be applied for the model with regime switching. In this paper, we develop a different method to overcome the above mentioned difficulty of defining $P(s, \alpha(s))$ in our model arisen in the proof of Theorem 5.2. We think that the definition of $P(s, \alpha(s))$ provides additional insight to our proof. In fact, if we interpret the solution of (5.11) as the flow of the initial state $(t, x, i)$ and define the matrix valued stochastic processes $X(s ; t, i)$ and $Y(s ; t, i)$ in (5.13), then we can prove that $Y(s ; t, i)=P(s, \alpha(s)) X(s ; t, i)$ under the definition of $P(t, i):=Y(t ; t, i), i \in \mathcal{S}$. Since $X(s ; t, i)$ is a.s. inverse at each $s \in[t, T]$, we finally derive the expression of $P(s, \alpha(s))=Y(s ; t, i) X(s ; t, i)^{-1}$. Furthermore, our method is more general and also can be applied to solving the problems in $[20,22]$ with random coefficients.

It is worthy to mention that $\mathrm{Lv}$ et al. [18] established the characterization of closed-loop optimal control when the coefficients are adapted processes with respect to the filtration generated by the Brownian motion, and Huang and $\mathrm{Yu}[5]$ derived some equivalent conditions for closed-loop optimal control for deterministic case. If there is only one regime jump state in our model, our characterization of the closed-loop optimal control becomes a special case of [18]. Furthermore, the equivalence between the uniform convex and the strongly regular solution of Riccati equation (Thm. 6.3) implies that $\widehat{R}$ is uniformly positive, which is the same as the statement (ii) of Theorem 4.6 of [5].

The first main contribution of our paper is to develop a new method for obtaining the representation of the cost functional for the stochastic LQ optimal control problem with regime switching jumps. In Sun et al. [20], the representation of the cost functional, which is the summary results of Yong and Zhou [30], is fundamental to prove the above equivalence. Unlike the techniques of analysis established in [20,30], our method for deriving the representation of the cost functional is mainly based on the technique of Itô's formula with jumps. The second main contribution of our paper is to adopt the stochastic flow theory for proving the equivalence between the closed-loop solvability and the existence of regular solution to the Riccati equation. Due to incorporating the regime switching jumps, the method applied in Sun et al. [20] no longer works for proving the equivalence between the closed-loop solvability and the existence of regular solution to the Riccati equation when one studies the stochastic LQ optimal control problem with regime switching jumps.

The rest of the paper is organized as follows. Section 2 introduces some useful notation, collect some preliminary results and state the stochastic LQ optimal control problem with regime switching jumps. Section 3 is devoted to deriving the representation of the cost functional using the technique of Itô's formula with jumps. In Sections 4 and 5, we prove the equivalence between the open-loop (closed-loop) solvability and the existence of an adapted solution to the corresponding FBSDE with constraint (i.e., the existence of a regular solution to the Riccati equation) for the stochastic LQ optimal control problem of Markovian regime switching system. The equivalence between the strongly regular solvability of the Riccati equation and the uniform convexity of the cost functional is established in Section 6. In the last section, we present an example which is open-loop solvable but not closed-loop solvable. 


\section{Preliminaries AND MOdel FORMUlation}

Let $(\Omega, \mathcal{F}, \mathbb{F}, \mathbb{P})$ be a complete filtered probability space on which a standard one-dimensional Brownian motion $W=\{W(t) ; 0 \leqslant t<\infty\}$ and a continuous time, finite-state, Markov chain $\alpha=\{\alpha(t) ; 0 \leqslant t<\infty\}$ are defined, where $\mathbb{F}=\left\{\mathcal{F}_{t}\right\}_{t \geqslant 0}$ is the natural filtration of $W$ and $\alpha$ augmented by all the $\mathbb{P}$-null sets in $\mathcal{F}$. In the rest of our paper, we use the following notation:

$\mathbb{N}$ : the set of natural numbers;

$\mathbb{R}_{+}, \overline{\mathbb{R}}_{+}:$the sets $[0, \infty)$ and $[0,+\infty]$ respectively;

$\mathbb{R}^{n}:$ the $n$-dimensional Euclidean space;

$M^{\top}$ : the transpose of any vector or matrix $M$;

$\operatorname{tr}[M]:$ the trace of a square matrix $M$;

$\mathcal{R}(M)$ : the range of the matrix $M$;

$\langle\cdot, \cdot\rangle:$ the inner products in possibly different Hilbert spaces;

$M^{\dagger}$ : the Moore-Penrose pseudo-inverse of the matrix $M$;

$\mathbb{R}^{n \times m}$ : the space of all $n \times m$ matrices endowed with the inner product

$$
\langle M, N\rangle \mapsto \operatorname{tr}\left[M^{\top} N\right] \text { and the norm }|M|=\sqrt{\operatorname{tr}\left[M^{\top} M\right]} ;
$$

$\mathbb{S}^{n}\left(\mathbb{S}_{+}^{n}\right):$ the set of all $n \times n$ symmetric (positive-definite) matrices.

Next, let $T>0$ be a fixed time horizon. For any $t \in[0, T)$ and Euclidean space $\mathbb{H}$, we set

$$
\begin{aligned}
C([t, T] ; \mathbb{H}) & =\{\varphi:[t, T] \rightarrow \mathbb{H} \mid \varphi(\cdot) \text { is continuous }\}, \\
L^{p}(t, T ; \mathbb{H}) & =\left\{\varphi:\left.[t, T] \rightarrow \mathbb{H}\left|\int_{t}^{T}\right| \varphi(s)\right|^{p} \mathrm{~d} s<\infty\right\}, \quad 1 \leqslant p<\infty, \\
L^{\infty}(t, T ; \mathbb{H}) & =\left\{\varphi:[t, T] \rightarrow \mathbb{H}\left|\begin{array}{l}
\operatorname{esssup} \\
s \in[t, T]
\end{array}\right| \varphi(s) \mid<\infty\right\},
\end{aligned}
$$

and

$$
\begin{aligned}
L_{\mathcal{F}_{T}}^{2}(\Omega ; \mathbb{H}) & =\left\{\xi: \Omega \rightarrow \mathbb{H} \mid \xi \text { is } \mathcal{F}_{T} \text {-measurable, } \mathbb{E}|\xi|^{2}<\infty\right\}, \\
L_{\mathbb{F}}^{2}(t, T ; \mathbb{H}) & =\left\{\varphi:[t, T] \times \Omega \rightarrow \mathbb{H} \mid \varphi(\cdot) \text { is } \mathbb{F} \text {-progressively measurable, } \mathbb{E} \int_{t}^{T}|\varphi(s)|^{2} \mathrm{~d} s<\infty\right\}, \\
L_{\mathbb{F}}^{2}(\Omega ; C([t, T] ; \mathbb{H})) & =\left\{\varphi:[t, T] \times \Omega \rightarrow \mathbb{H} \mid \varphi(\cdot) \text { is } \mathbb{F} \text {-adapted, continuous, } \mathbb{E}\left[\sup _{s \in[t, T]}|\varphi(s)|^{2}\right]<\infty\right\}, \\
L_{\mathbb{F}}^{2}\left(\Omega ; L^{1}(t, T ; \mathbb{H})\right) & =\left\{\varphi:[t, T] \times \Omega \rightarrow \mathbb{H} \mid \varphi(\cdot) \text { is } \mathbb{F} \text {-progressively measurable, } \mathbb{E}\left(\int_{t}^{T}|\varphi(s)| \mathrm{d} s\right)^{2}<\infty\right\} .
\end{aligned}
$$

For an $\mathbb{S}^{n}$-valued function $F(\cdot)$ on $[t, T]$, we use the notation $F(\cdot) \gg 0$ to indicate that $F(\cdot)$ is uniformly positive definite on $[t, T]$, i.e., there exists a constant $\delta>0$ such that

$$
F(s) \geqslant \delta I, \quad \text { a.e. } s \in[t, T]
$$


Now we start to formulate our system. We identify the state space of the chain $\alpha$ with a finite set $\mathcal{S}:=$ $\{1,2 \ldots, D\}$, where $D \in \mathbb{N}$. Without loss of generality, we assume that the Markov chain is irreducible. To specify the statistical or probabilistic properties of the chain $\alpha$, we define the generator $\lambda(t):=\left[\lambda_{i j}(t)\right]_{i, j=1,2, \ldots, D}$ of the chain under $\mathbb{P}$. Here, for each $i, j=1,2, \ldots, D, \lambda_{i j}(t)$ is the constant transition intensity of the chain from state $i$ to state $j$ at time $t$. Note that $\lambda_{i j}(t) \geq 0$, for $i \neq j$ and $\sum_{j=1}^{D} \lambda_{i j}(t)=0$, so $\lambda_{i i}(t) \leq 0$. In what follows, for each $i, j=1,2, \ldots, D$ with $i \neq j$, we suppose that $\lambda_{i j}(t)>0$, so $\lambda_{i i}(t)<0$. For each fixed $j=1,2, \ldots, D$, let $N_{i j}(t)$ be the number of jumps from state $i$ into state $j$ up to time $t$ and set

$$
\widetilde{N}_{i i}(t) \equiv 0, \quad \widetilde{N}_{i j}(t):=N_{i j}(t)-\tilde{\lambda}_{i j}(t), \quad i \neq j
$$

with $\tilde{\lambda}_{i j}(t):=\int_{0}^{t} \lambda_{i j}(s) I_{\{\alpha(s-)=i\}} \mathrm{d} s$. Then for each $i, j=1,2, \ldots, D$, the term $\widetilde{N}_{i j}(t)$ is an $(\mathbb{F}, \mathbb{P})$-martingale.

Consider the following controlled Markovian regime switching linear stochastic differential equation on the finite horizon $[t, T]$ :

$$
\left\{\begin{aligned}
& \mathrm{d} X^{u}(s ; t, x, i)=\left[A(s, \alpha(s)) X^{u}(s ; t, x, i)+B(s, \alpha(s)) u(s)+b(s)\right] \mathrm{d} s \\
&+\left[C(s, \alpha(s)) X^{u}(s ; t, x, i)+D(s, \alpha(s)) u(s)+\sigma(s)\right] \mathrm{d} W(s), \quad s \in[t, T], \\
& X^{u}(t ; t, x, i)=x, \quad \alpha(t)=i,
\end{aligned}\right.
$$

where $A(\cdot, \cdot), B(\cdot, \cdot), C(\cdot, \cdot), D(\cdot, \cdot)$ are given deterministic matrix-valued functions of proper dimensions, and $b(\cdot), \sigma(\cdot)$ are vector-valued $\mathbb{F}$-progressively measurable processes. In the above, $X^{u}(\cdot ; t, x, i)$, valued in $\mathbb{R}^{n}$, is the state process, and $u(\cdot)$, valued in $\mathbb{R}^{m}$, is the control process. $u(\cdot)$ is called an admissible control on $[t, T]$, if it belongs to the following Hilbert space:

$$
\mathcal{U}[t, T]=\left\{u:[t, T] \times \Omega \rightarrow \mathbb{R}^{m} \mid u(\cdot) \text { is } \mathbb{F} \text {-progressively measurable, } \mathbb{E} \int_{t}^{T}|u(s)|^{2} \mathrm{~d} s<\infty\right\}
$$

For any admissible control $u(\cdot)$, we consider the following general quadratic cost functional:

$$
\begin{aligned}
J(t, x, i ; u(\cdot)) \triangleq \mathbb{E}\{\langle & \left.G(T, \alpha(T)) X^{u}(T ; t, x, i)+2 g(T, \alpha(T)), X^{u}(T ; t, x, i)\right\rangle \\
& +\int_{t}^{T}\left[\left\langle Q(s, \alpha(s)) X^{u}(s ; t, x, i)+2 q(s, \alpha(s)), X^{u}(s ; t, x, i)\right\rangle\right. \\
& \left.\left.+2\left\langle S(s, \alpha(s)) X^{u}(s ; t, x, i), u(s)\right\rangle+\langle R(s, \alpha(s)) u(s)+2 \rho(s, \alpha(s)), u(s)\rangle\right] \mathrm{d} s\right\},
\end{aligned}
$$

where $G(T, i)$ is a symmetric matrix, $Q(\cdot, i), S(\cdot, i), R(\cdot, i), i=1,2, \ldots, D$ are deterministic matrix-valued functions of proper dimensions with $Q(\cdot, i)^{\top}=Q(\cdot, i), R(\cdot, i)^{\top}=R(\cdot, i) ; g(T, \cdot)$ is allowed to be an $\mathcal{F}_{T}$-measurable random variable and $q(\cdot, \cdot), \rho(\cdot, \cdot)$ are allowed to be vector-valued $\mathbb{F}$-progressively measurable processes.

The following standard assumptions will be in force throughout this paper.

(H1) The coefficients of the state equation satisfy the following: for each $i \in \mathcal{S}$,

$$
\left\{\begin{array}{lll}
A(\cdot, i) \in L^{1}\left(0, T ; \mathbb{R}^{n \times n}\right), & B(\cdot, i) \in L^{2}\left(0, T ; \mathbb{R}^{n \times m}\right), \quad b(\cdot) \in L_{\mathbb{F}}^{2}\left(\Omega ; L^{1}\left(0, T ; \mathbb{R}^{n}\right)\right), \\
C(\cdot, i) \in L^{2}\left(0, T ; \mathbb{R}^{n \times n}\right), & D(\cdot, i) \in L^{\infty}\left(0, T ; \mathbb{R}^{n \times m}\right), \quad \sigma(\cdot) \in L_{\mathbb{F}}^{2}\left(0, T ; \mathbb{R}^{n}\right) .
\end{array}\right.
$$


(H2) The weighting coefficients in the cost functional satisfy the following: for each $i \in \mathcal{S}$

$$
\left\{\begin{array}{l}
G(T, i) \in \mathbb{S}^{n}, \quad Q(\cdot, i) \in L^{1}\left(0, T ; \mathbb{S}^{n}\right), \quad S(\cdot, i) \in L^{2}\left(0, T ; \mathbb{R}^{m \times n}\right), \quad R(\cdot, i) \in L^{\infty}\left(0, T ; \mathbb{S}^{m}\right), \\
g(T, i) \in L_{\mathcal{F}_{T}}^{2}\left(\Omega ; \mathbb{R}^{n}\right), \quad q(\cdot, i) \in L_{\mathbb{F}}^{2}\left(\Omega ; L^{1}\left(0, T ; \mathbb{R}^{n}\right)\right), \quad \rho(\cdot, i) \in L_{\mathbb{F}}^{2}\left(0, T ; \mathbb{R}^{m}\right) .
\end{array}\right.
$$

Now we state the stochastic LQ optimal control problem for the Markovian regime switching system as follows.

Problem 2.1. (M-SLQ) For any given initial pair $(t, x, i) \in[0, T) \times \mathbb{R}^{n} \times \mathcal{S}$, find a $u^{*}(\cdot) \in \mathcal{U}[t, T]$, such that

$$
J\left(t, x, i ; u^{*}(\cdot)\right)=\inf _{u(\cdot) \in \mathcal{U}[t, T]} J(t, x, i ; u(\cdot)) \triangleq V(t, x, i) .
$$

Any $u^{*}(\cdot) \in \mathcal{U}[t, T]$ satisfying $(2.3)$ is called an optimal control of Problem (M-SLQ) for the initial pair $(t, x, i)$, and the corresponding path $X^{*}(\cdot) \equiv X^{u^{*}}(\cdot ; t, x, i)$ is called an optimal state process; the pair $\left(X^{*}(\cdot), u^{*}(\cdot)\right)$ is called an optimal pair. The function $V(\cdot, \cdot, \cdot)$ is called the value function of Problem (M-SLQ). When $b(\cdot), \sigma(\cdot), g(T, \cdot), q(\cdot, \cdot), \rho(\cdot, \cdot)=0$, we denote the corresponding Problem (M-SLQ) by Problem (M-SLQ) ${ }^{0}$. The corresponding cost functional and value function are denoted by $J^{0}(t, x, i ; u(\cdot))$ and $V^{0}(t, x, i)$, respectively.

Similar to Sun et al. [20], we introduce the following definitions of open-loop (closed-loop) optimal control.

Definition 2.2. (Open-loop) For given $t \in[0, T]$, an element $u^{*}(\cdot) \in \mathcal{U}[t, T]$ is called an open-loop optimal control of Problem (M-SLQ) for the initial pair $(t, x, i) \in[0, T] \times \mathbb{R}^{n} \times \mathcal{S}$ if

$$
J\left(t, x, i ; u^{*}(\cdot)\right) \leqslant J(t, x, i ; u(\cdot)), \quad \forall u(\cdot) \in \mathcal{U}[t, T] .
$$

Definition 2.3. (Closed-loop) For given $t \in[0, T]$, let $\Theta(\cdot, \cdot):[t, T] \times \mathcal{S} \rightarrow \mathbb{R}^{m \times n}$ be a deterministic function and $v(\cdot):[t, T] \times \Omega \rightarrow \mathbb{R}^{m}$ be an $\mathbb{F}$-progressively measurable process.

(i) We call $(\Theta(\cdot, \cdot), v(\cdot))$ a closed-loop strategy on $[t, T]$ if

$$
\mathbb{E} \int_{t}^{T}|\Theta(s, \alpha(s))|^{2} \mathrm{~d} s<\infty \text { and } v(\cdot) \in \mathcal{U}[t, T] .
$$

The set of all closed-loop strategies $(\Theta(\cdot, \cdot), v(\cdot))$ on $[t, T]$ is denoted by $\mathcal{G}[t, T]$.

(ii) A pair $\left(\Theta^{*}(\cdot, \cdot), v^{*}(\cdot)\right) \in \mathcal{G}[t, T]$ is called a closed-loop optimal strategy of Problem (M-SLQ) on $[t, T]$ if

$$
J\left(t, x, i ; \Theta^{*}(\cdot, \alpha(\cdot)) X^{*}(\cdot)+v^{*}(\cdot)\right) \leqslant J(t, x, i ; u(\cdot)), \quad \forall(x, i) \in \mathbb{R}^{n} \times \mathcal{S}, u(\cdot) \in \mathcal{U}[t, T],
$$

where $X^{*}(\cdot)$ is the strong solution to the following closed-loop system:

$$
\left\{\begin{aligned}
\mathrm{d} X^{*}(s)= & \left\{\left[A(s, \alpha(s))+B(s, \alpha(s)) \Theta^{*}(s, \alpha(s))\right] X^{*}(s)+B(s, \alpha(s)) v^{*}(s)+b(s)\right\} \mathrm{d} s \\
& +\left\{\left[C(s, \alpha(s))+D(s, \alpha(s)) \Theta^{*}(s, \alpha(s))\right] X^{*}(s)+D(s, \alpha(s)) v^{*}(s)+\sigma(s)\right\} \mathrm{d} W(s), \\
X^{*}(t)= & x, \quad \alpha(t)=i
\end{aligned}\right.
$$

Remark 2.4. (i) The inequality (2.5) in defining the closed-loop optimal strategy can be replaced by the following inequality:

$$
J\left(t, x, i ; \Theta^{*}(\cdot, \alpha(\cdot)) X^{*}(\cdot)+v^{*}(\cdot)\right) \leq J(t, x, i ; \Theta(\cdot, \alpha(\cdot)) X(\cdot)+v(\cdot)), \forall(x, i) \in \mathbb{R}^{n} \times \mathcal{S},(\Theta(\cdot, \cdot), v(\cdot)) \in \mathcal{G}[t, T] .
$$


In fact, using the similar method of Sun and Yong [23] (see Prop. 2.1.5), we can prove the equivalence of (2.5) and (2.7). Therefore, we can take (2.7) for defining the closed-loop optimal strategy.

(ii) We emphasize that in the definition of closed-loop optimal strategy, $(2.5)$ must be true for all $(x, i) \in$ $\mathbb{R}^{n} \times \mathcal{S}$. One sees that if $\left(\Theta^{*}(\cdot, \alpha(\cdot)), v^{*}(\cdot)\right)$ is a closed-loop optimal strategy of problem (M-SLQ) on $[t, T]$, then the outcome $u^{*}(\cdot) \equiv \Theta^{*}(\cdot, \alpha(\cdot)) X^{*}(\cdot)+v^{*}(\cdot)$ is an open-loop optimal control of Problem (M-SLQ) for the initial pair $\left(t, X^{*}(t), \alpha(t)\right)$. Hence, the existence of closed-loop optimal strategies implies the existence of open-loop optimal controls. However, the existence of open-loop optimal controls does not necessarily imply the existence of a closed-loop optimal strategy, see an example in section 7 for more details.

To simplify the notation of our further analysis, we introduce the following forward-backward stochastic differential equation (FBSDE for short) on a finite horizon $[t, T]$ :

$$
\left\{\begin{aligned}
\mathrm{d} X^{u}(s ; t, x, i)= & {\left[A(s, \alpha(s)) X^{u}(s ; t, x, i)+B(s, \alpha(s)) u(s)+b(s)\right] \mathrm{d} s } \\
& +\left[C(s, \alpha(s)) X^{u}(s ; t, x, i)+D(s, \alpha(s)) u(s)+\sigma(s)\right] \mathrm{d} W(s), \\
\mathrm{d} Y^{u}(s ; t, x, i)= & -\left[A(s, \alpha(s))^{\top} Y^{u}(s ; t, x, i)+C(s, \alpha(s))^{\top} Z^{u}(s ; t, x, i)\right. \\
& \left.+Q(s, \alpha(s)) X^{u}(s ; t, x, i)+S(s, \alpha(s))^{\top} u(s)+q(s, \alpha(s))\right] \mathrm{d} s \\
& +Z^{u}(s ; t, x, i) \mathrm{d} W(s)+\sum_{l, k=1}^{D} \Gamma_{l k}^{u}(s ; t, x, i) \mathrm{d} \widetilde{N}_{l k}(s), \quad s \in[t, T], \\
X^{u}(t ; t, x, i)= & x, \quad \alpha(t)=i, \quad Y^{u}(T ; t, x, i)=G(T, \alpha(T)) X^{u}(T ; t, x, i)+g(T, \alpha(T)) .
\end{aligned}\right.
$$

The solution of the above FBSDE system is denoted by $\left(X^{u}(\cdot ; t, x, i), Y^{u}(\cdot ; t, x, i), Z^{u}(\cdot ; t, x, i), \Gamma^{u}(\cdot ; t, x, i)\right)$, where $\Gamma^{u}(\cdot ; t, x, i):=\left(\Gamma_{l k}^{u}(\cdot ; t, x, i)\right)_{l, k=1}^{D}$. If the control $u(\cdot)$ is chosen as $\Theta(\cdot, \alpha(\cdot)) X(\cdot)+v(\cdot)$, we use the notation $\left(X^{\Theta, v}(\cdot ; t, x, i), Y^{\Theta, v}(\cdot ; t, x, i), Z^{\Theta, v}(\cdot ; t, x, i), \Gamma^{\Theta, v}(\cdot ; t, x, i)\right)$ denoting the solution of the above FBSDE. If $b(\cdot)=$ $\sigma(\cdot)=q(\cdot, \cdot)=g(\cdot, \cdot)=0$, the solution of the above FBSDE system is denoted by

$$
\left(X_{0}^{u}(\cdot ; t, x, i), Y_{0}^{u}(\cdot ; t, x, i), Z_{0}^{u}(\cdot ; t, x, i), \Gamma_{0}^{u}(\cdot ; t, x, i)\right) .
$$

\section{Representation of the COST FUnCtional}

In this section, we present a representation of the cost functional for Problem (M-SLQ), which plays a crucial role in the study of open-loop/closed-loop solvability of Problem (M-SLQ). Unlike the method adopted in Yong and Zhou [30], we derive the representation of the cost functional using the technique of Itô's formula with jumps.

Proposition 3.1. Let $(\mathrm{H} 1)-(\mathrm{H} 2)$ hold and $\left(X^{u}(\cdot ; t, x, i), Y^{u}(\cdot ; t, x, i), Z^{u}(\cdot ; t, x, i), \Gamma^{u}(\cdot ; t, x, i)\right)$ be the solution of (2.8). Then for $(t, x, i) \in[0, T] \times \mathbb{R}^{n} \times \mathcal{S}$ and $u(\cdot) \in \mathcal{U}[t, T]$,

$$
\begin{aligned}
J^{0}(t, x, i ; u(\cdot)) & =\left\langle M_{2}(t, i) u, u\right\rangle+2\left\langle M_{1}(t, i) x, u\right\rangle+\left\langle M_{0}(t, i) x, x\right\rangle, \\
J(t, x, i ; u(\cdot)) & =\left\langle M_{2}(t, i) u, u\right\rangle+2\left\langle M_{1}(t, i) x, u\right\rangle+\left\langle M_{0}(t, i) x, x\right\rangle+2\left\langle\nu_{t}, u\right\rangle+2\left\langle y_{t}, x\right\rangle+c_{t},
\end{aligned}
$$

where

$$
\begin{aligned}
M_{0}(t, i) x= & \mathbb{E}\left[Y_{0}^{0}(t ; t, x, i)\right], \\
\left(M_{1}(t, i) x\right)(s)= & B(s, \alpha(s))^{\top} Y_{0}^{0}(s ; t, x, i)+D(s, \alpha(s))^{\top} Z_{0}^{0}(s ; t, x, i) \\
& +S(s, \alpha(s)) X_{0}^{0}(s ; t, x, i), \quad s \in[t, T], \\
\left(M_{2}(t, i) u\right)(s)= & B(s, \alpha(s))^{\top} Y_{0}^{u}(s ; t, 0, i)+D(s, \alpha(s))^{\top} Z_{0}^{u}(s ; t, 0, i) \\
& +S(s, \alpha(s)) X_{0}^{u}(s ; t, 0, i)+R(s, \alpha(s)) u(s), \quad s \in[t, T],
\end{aligned}
$$




$$
\begin{aligned}
y_{t}= & \mathbb{E}\left[Y^{0}(t ; t, 0, i)\right], \\
\nu_{t}(s)= & {[B(s, \alpha(s))]^{\top} Y^{0}(s ; t, 0, i)+D(s, \alpha(s))^{\top} Z^{0}(s ; t, 0, i) } \\
& +S(s, \alpha(s)) X^{0}(s ; t, 0, i)+\rho(s, \alpha(s)), \quad s \in[t, T], \\
c_{t}= & \mathbb{E}\left[\left\langle G(T, \alpha(T)) X^{0}(T ; t, 0, i)+2 g(T, \alpha(T)), X^{0}(T ; t, 0, i)\right\rangle\right. \\
& \left.+\int_{t}^{T}\left\langle Q(s, \alpha(s)) X^{0}(s ; t, 0, i)+2 q(s, \alpha(s)), X^{0}(s ; t, 0, i)\right\rangle \mathrm{d} s\right] .
\end{aligned}
$$

Proof. Let

$$
\begin{aligned}
& I_{1}:=\mathbb{E} {\left[\left\langle G(T, \alpha(T)) X_{0}^{u}(T ; t, x, i), X_{0}^{u}(T ; t, x, i)\right\rangle\right] } \\
& I_{2}:=\mathbb{E}\left\{\int _ { t } ^ { T } \left[\left\langle Q(s, \alpha(s)) X_{0}^{u}(s ; t, x, i), X_{0}^{u}(s ; t, x, i)\right\rangle\right.\right. \\
&\left.\left.\quad+2\left\langle S(s, \alpha(s)) X_{0}^{u}(s ; t, x, i), u(s)\right\rangle+\langle R(s, \alpha(s)) u(s), u(s)\rangle\right] \mathrm{d} s\right\}
\end{aligned}
$$

then we have $J^{0}(t, x, i ; u(\cdot))=I_{1}+I_{2}$. Observing

$$
X_{0}^{u}(\cdot ; t, x, i)=X_{0}^{u}(\cdot ; t, 0, i)+X_{0}^{0}(\cdot ; t, x, i)
$$

therefore

$$
\begin{aligned}
& I_{1}=\mathbb{E}[\langle\left.G(T, \alpha(T)) X_{0}^{u}(T ; t, 0, i), X_{0}^{u}(T ; t, 0, i)\right\rangle, \\
&\left.+2\left\langle G(T, \alpha(T)) X_{0}^{0}(T ; t, x, i), X_{0}^{u}(T ; t, 0, i)\right\rangle+\left\langle G(T, \alpha(T)) X_{0}^{0}(T ; t, x, i), X_{0}^{0}(T ; t, x, i)\right\rangle\right], \\
& I_{2}=\mathbb{E}\left\{\int _ { t } ^ { T } \left[\left\langle Q(s, \alpha(s)) X_{0}^{u}(s ; t, 0, i), X_{0}^{u}(s ; t, 0, i)\right\rangle+\left\langle Q(s, \alpha(s)) X_{0}^{0}(s ; t, x, i), X_{0}^{0}(s ; t, x, i)\right\rangle\right.\right. \\
&+2\left\langle Q(s, \alpha(s)) X_{0}^{0}(s ; t, x, i), X_{0}^{u}(s ; t, 0, i)\right\rangle+2\left\langle S(s, \alpha(s)) X_{0}^{u}(s ; t, 0, i), u(s)\right\rangle \\
&\left.\left.+2\left\langle S(s, \alpha(s)) X_{0}^{0}(s ; t, x, i), u(s)\right\rangle+\langle R(s, \alpha(s)) u(s), u(s)\rangle\right] \mathrm{d} s\right\} .
\end{aligned}
$$

Applying Itô's formula to $\left\langle Y_{0}^{u}(s ; t, 0, i), X_{0}^{u}(s ; t, 0, i)\right\rangle,\left\langle Y_{0}^{0}(s ; t, x, i), X_{0}^{u}(s ; t, 0, i)\right\rangle$ and $\left\langle Y_{0}^{0}(s ; t, x, i), X_{0}^{0}(s ; t, x, i)\right\rangle$, we obtain

$$
\begin{aligned}
J^{0}(t, x, i ; u(\cdot)) & =I_{1}+I_{2} \\
& =\mathbb{E} \int_{t}^{T}\left\langle\left(M_{2}(t, i) u\right)(s), u(s)\right\rangle \mathrm{d} s+2 \mathbb{E} \int_{t}^{T}\left\langle\left(M_{1}(t, i) x\right)(s), u(s)\right\rangle \mathrm{d} s+\left\langle\mathbb{E}\left[Y_{0}^{0}(t ; t, x, i)\right], x\right\rangle \\
& =\left\langle M_{2}(t, i) u, u\right\rangle+2\left\langle M_{1}(t, i) x, u\right\rangle+\left\langle M_{0}(t, i) x, x\right\rangle .
\end{aligned}
$$

Let

$$
I_{3}:=\mathbb{E}\left[\left\langle G(T, \alpha(T)) X^{u}(T ; t, x, i)+2 g(T, \alpha(T)), X^{u}(T ; t, x, i)\right\rangle\right]
$$




$$
\begin{aligned}
I_{4}:=\mathbb{E}\left\{\int_{t}^{T}[\langle\right. & \left.Q(s, \alpha(s)) X^{u}(s ; t, x, i)+2 q(s, \alpha(s)), X^{u}(s ; t, x, i)\right\rangle \\
& \left.\left.+2\left\langle S(s, \alpha(s)) X^{u}(s ; t, x, i), u(s)\right\rangle+\langle R(s, \alpha(s)) u(s)+2 \rho(s, \alpha(s)), u(s)\rangle\right] \mathrm{d} s\right\},
\end{aligned}
$$

then $J(t, x, i ; u(\cdot))=I_{3}+I_{4}$. Observing

$$
X^{u}(\cdot ; t, x, i)=X_{0}^{u}(\cdot ; t, x, i)+X^{0}(\cdot ; t, 0, i)
$$

therefore

$$
I_{3}=I_{31}+I_{32}+I_{33}, \quad I_{4}=I_{41}+I_{42}+I_{43},
$$

where

$$
\begin{aligned}
& I_{31}:=\mathbb{E}\left\langle G(T, \alpha(T)) X_{0}^{u}(T ; t, x, i), X_{0}^{u}(T ; t, x, i)\right\rangle, \\
& I_{32}:=2 \mathbb{E}\left\langle G(T, \alpha(T)) X^{0}(T ; t, 0, i)+g(T, \alpha(T)), X_{0}^{u}(T ; t, x, i)\right\rangle, \\
& I_{33}:=\mathbb{E}\left\langle G(T, \alpha(T)) X^{0}(T ; t, 0, i)+2 g(T, \alpha(T)), X^{0}(T ; t, 0, i),\right. \\
& I_{41}:=\mathbb{E} \int_{t}^{T}\left[\left\langle Q(s, \alpha(s)) X_{0}^{u}(s ; t, x, i), X_{0}^{u}(s ; t, x, i)\right\rangle\right. \\
& \left.\quad+2\left\langle S(s, \alpha(s)) X_{0}^{u}(s ; t, x, i), u(s)\right\rangle+\langle R(s, \alpha(s)) u(s), u(s)\rangle\right] \mathrm{d} s, \\
& \begin{aligned}
I_{42}:=2 \mathbb{E} \int_{t}^{T}\left[\left\langle Q(s, \alpha(s)) X^{0}(s ; t, 0, i)+q(s, \alpha(s)), X_{0}^{u}(s ; t, x, i)\right\rangle\right. \\
\left.\quad+2\left\langle S(s, \alpha(s)) X^{0}(s ; t, 0, i)+\rho(s, \alpha(s)), u(s)\right\rangle\right] \mathrm{d} s, \\
I_{43}:=\mathbb{E} \int_{t}^{T}\left[\left\langle Q(s, \alpha(s)) X^{0}(s ; t, 0, i)+2 q(s, \alpha(s)), X^{0}(s ; t, 0, i)\right\rangle\right] \mathrm{d} s .
\end{aligned}
\end{aligned}
$$

Applying Itô's formula to $\left\langle Y^{0}(s ; t, 0, i), X_{0}^{u}(s ; t, x, i)\right\rangle$ yields

$$
I_{32}+I_{42}=2\left\langle\mathbb{E} Y^{0}(t ; t, 0, i), x\right\rangle+2 \mathbb{E} \int_{t}^{T}\left\langle\nu_{t}(s), u(s)\right\rangle \mathrm{d} s=2\left\langle y_{t}, x\right\rangle+2\left\langle\nu_{t}, u\right\rangle .
$$

Noting that $J^{0}(t, x, i ; u(\cdot))=I_{31}+I_{41}, c_{t}=I_{33}+I_{43}$, we have

$$
\begin{aligned}
J(t, x, i ; u(\cdot)) & =I_{3}+I_{4}=\left(I_{31}+I_{41}\right)+\left(I_{32}+I_{42}\right)+\left(I_{33}+I_{43}\right) \\
& =\left\langle M_{2}(t, i) u, u\right\rangle+2\left\langle M_{1}(t, i) x, u\right\rangle+\left\langle M_{0}(t, i) x, x\right\rangle+2\left\langle\nu_{t}, u\right\rangle+2\left\langle y_{t}, x\right\rangle+c_{t} .
\end{aligned}
$$

Next, we show that the above characterizations of operators $M_{0}(t, i)$ and $M_{2}(t, i)$ is equivalent to the results obtained by the technique of functional analysis. 
Proposition 3.2. For $i=1,2, \ldots, D, M_{0}(\cdot, i)$ defined in 3.1 admits the following Feynman-Kac representation:

$$
M_{0}(t, i)=\mathbb{E}\left[\Phi(T ; t, i)^{\top} G(T, \alpha(T)) \Phi(T ; t, i)+\int_{t}^{T} \Phi(s ; t, i)^{\top} Q(s, \alpha(s)) \Phi(s ; t, i) \mathrm{d} s\right],
$$

where $\Phi(\cdot ; t, i)$ is the solution to the following $S D E$ for $\mathbb{R}^{n \times n}$-valued process:

$$
\left\{\begin{aligned}
d \Phi(s ; t, i) & =A(s, \alpha(s)) \Phi(s ; t, i) \mathrm{d} s+C(s, \alpha(s)) \Phi(s ; t, i) \mathrm{d} W(s), \quad s \in[t, T], \\
\Phi(t ; t, i) & =I, \quad \alpha(t)=i .
\end{aligned}\right.
$$

Furthermore, $M_{0}(t, i)$ also solves the following ordinary differential equations

$$
\left\{\begin{aligned}
\dot{M}_{0}(t, i) & +M_{0}(t, i) A(t, i)+A(t, i)^{\top} M_{0}(t, i) \\
& +C(t, i)^{\top} M_{0}(t, i) C(t, i)+Q(t, i)+\sum_{k=1}^{D} \lambda_{i k}(t) M_{0}(t, k)=0, \quad(t, i) \in[0, T] \times \mathcal{S}, \\
M_{0}(T, i) & =G(T, i), \quad i \in \mathcal{S} .
\end{aligned}\right.
$$

Proof. Let $\Phi(\cdot ; t, i)$ be the solution to $(3.6)$. Then it is easy to verify that

$$
X_{0}^{0}(s ; t, x, i)=\Phi(s ; t, i) x .
$$

Applying Itô's formula to $\left\langle Y_{0}^{0}(s ; t, x, i), X_{0}^{0}(s ; t, x, i)\right\rangle$, we can easily obtain

$$
\begin{aligned}
& \mathbb{E}\left[\left\langle G(T, \alpha(T)) X_{0}^{0}(T ; t, x, i), X_{0}^{0}(T ; t, x, i)\right\rangle\right] \\
& =\left\langle\mathbb{E}\left[Y_{0}^{0}(t ; t, x, i)\right], x\right\rangle-\mathbb{E}\left[\int_{t}^{T} X_{0}^{0}(s ; t, x, i)^{\top} Q(s, \alpha(s)) X_{0}^{0}(s ; t, x, i) \mathrm{d} s\right] .
\end{aligned}
$$

Therefore,

$$
\begin{aligned}
&\left\langle\mathbb{E}\left[Y_{0}^{0}(t ; t, x, i)\right], x\right\rangle=\mathbb{E}\left[\left\langle G(T, \alpha(T)) X_{0}^{0}(T ; t, x, i), X_{0}^{0}(T ; t, x, i)\right\rangle\right]+\mathbb{E}\left[\int_{t}^{T} X_{0}^{0}(s ; t, x, i)^{\top} Q(s, \alpha(s)) X_{0}^{0}(s ; t, x, i) \mathrm{d} s\right] \\
&=\mathbb{E}[\langle G(T, \alpha(T)) \Phi(T ; t, i) x, \Phi(T ; t, i) x\rangle] \quad+\mathbb{E}\left[\int_{t}^{T} x^{\top} \Phi(s ; t, i)^{\top} Q(s, \alpha(s)) \Phi(s ; t, i) x \mathrm{~d} s\right] \\
&=\mathbb{E}\left[\left\langle\Phi(T ; t, i)^{\top} G(T, \alpha(T)) \Phi(T ; t, i) x, x\right\rangle\right] \quad+\mathbb{E}\left[\int_{t}^{T}\left\langle\Phi(s ; t, i)^{\top} Q(s, \alpha(s)) \Phi(s ; t, i) x, x\right\rangle \mathrm{d} s\right] \\
&=\left\langle\mathbb{E}\left[\Phi(T ; t, i)^{\top} G(T, \alpha(T)) \Phi(T ; t, i)+\int_{t}^{T} \Phi(s ; t, i)^{\top} Q(s, \alpha(s)) \Phi(s ; t, i) \mathrm{d} s\right] x, x\right\rangle .
\end{aligned}
$$

Noting $M_{0}(t, i) x=\mathbb{E}\left[Y_{0}^{0}(t ; t, x, i)\right]$, we have

$$
M_{0}(t, i)=\mathbb{E}\left[\Phi(T ; t, i)^{\top} G(T, \alpha(T)) \Phi(T ; t, i)+\int_{t}^{T} \Phi(s ; t, i)^{\top} Q(s, \alpha(s)) \Phi(s ; t, i) \mathrm{d} s\right] .
$$


Suppose that $\widetilde{M}(\cdot, i)$ satisfy ODE $(3.7)$. Next we prove that $\widetilde{M}(\cdot, i)=M_{0}(\cdot, i)$. Observing that

$$
d \widetilde{M}(s, \alpha(s))=\dot{\widetilde{M}}(s, \alpha(s)) \mathrm{d} s+\sum_{l, k=1}^{D}[\widetilde{M}(s, k)-\widetilde{M}(s, l)] d \lambda_{l k}(s)+\sum_{l, k=1}^{D}[\widetilde{M}(s, k)-\widetilde{M}(s, l)] d \widetilde{N}_{l k}(s) .
$$

Hence, applying Itô's formula to $\Phi(s ; t, i)^{\top} \widetilde{M}(s, \alpha(s)) \Phi(s ; t, i)$ leads to

$$
\begin{aligned}
\widetilde{M}(t, i) & =\mathbb{E}\left[\Phi(T ; t, i)^{\top} G(T, \alpha(T)) \Phi(T ; t, i)+\int_{t}^{T} \Phi(s ; t, i)^{\top} Q(s, \alpha(s)) \Phi(s ; t, i) \mathrm{d} s\right] \\
& =M_{0}(t, i) .
\end{aligned}
$$

Thus we complete our proof.

Proposition 3.3. The operator $M_{2}(\cdot, i)$ defined in 3.1 admits the following representation:

$$
M_{2}(t, i)=\widehat{L}_{t}^{*} G(T, \alpha(T)) \widehat{L}_{t}+L_{t}^{*} Q(\cdot, \alpha(\cdot)) L_{t}+S(\cdot, \alpha(\cdot)) L_{t}+L_{t}^{*} S(\cdot, \alpha(\cdot))^{\top}+R(\cdot, \alpha(\cdot)),
$$

where the operators

$$
L_{t}: \mathcal{U}[t, T] \rightarrow L_{\mathbb{F}}^{2}\left(t, T ; \mathbb{R}^{n}\right), \quad \widehat{L}_{t}: \mathcal{U}[t, T] \rightarrow L_{\mathbb{F}_{T}}^{2}\left(\Omega ; \mathbb{R}^{n}\right)
$$

are defined as follows:

$$
\begin{aligned}
\left(L_{t} u\right)(\cdot)=\Phi(\cdot ; t, i)\left\{\int_{t} \Phi(r ; t, i)^{-1}[B(r, \alpha(r))-C(r, \alpha(r)) D(r, \alpha(r))] u(r) \mathrm{d} r\right. \\
\left.\quad+\int_{t} \Phi(r ; t, i)^{-1} D(r, \alpha(r)) u(r) \mathrm{d} W(r)\right\} \\
\widehat{L}_{t} u=\left(L_{t} u\right)(T),
\end{aligned}
$$

and $L_{t}^{*}$ and $\widehat{L}_{t}^{*}$ are the adjoint operators of $L_{t}$ and $\widehat{L}_{t}$, respectively.

Proof. Noting that the solution $X_{0}^{u}(\cdot ; t, 0, i)$ of $(2.8)$ can be written as follows:

$$
\begin{aligned}
X_{0}^{u}(s ; t, 0, i)= & \Phi(s ; t, i)\left\{\int_{t}^{s} \Phi(r ; t, i)^{-1}[B(r, \alpha(r))-C(r, \alpha(r)) D(r, \alpha(r))] u(r) \mathrm{d} r\right. \\
& \left.+\int_{t}^{s} \Phi(r ; t, i)^{-1} D(r, \alpha(r)) u(r) \mathrm{d} W(r)\right\} \\
= & \left(L_{t} u\right)(s) .
\end{aligned}
$$

Applying Itô's formula to $\left\langle Y_{0}^{u}(s ; t, 0, i), X_{0}^{u}(s ; t, 0, i)\right\rangle$ yields

$$
\begin{aligned}
&\left\langle\left(M_{2}(t, i)\right) u, u\right\rangle=\mathbb{E}\left\{\left\langle G(T, \alpha(T)) X_{0}^{u}(T ; t, 0, i), X_{0}^{u}(T ; t, 0, i)\right\rangle\right. \\
&+\int_{t}^{T}\left[\left\langle Q(s, \alpha(s)) X_{0}^{u}(s ; t, 0, i), X_{0}^{u}(s ; t, 0, i)\right\rangle+\left\langle S(s, \alpha(s)) X_{0}^{u}(s ; t, 0, i), u(s)\right\rangle\right. \\
&\left.\left.+\left\langle S(s, \alpha(s))^{\top} u(s), X_{0}^{u}(s ; t, 0, i)\right\rangle+\langle R(s, \alpha(s)) u(s), u(s)\rangle\right] \mathrm{d} s\right\}
\end{aligned}
$$




$$
\begin{aligned}
= & \mathbb{E}\left[\left\langle G(T, \alpha(T)) \widehat{L}_{t} u, \widehat{L}_{t} u\right\rangle\right]+\left\langle Q(\cdot, \alpha(\cdot)) L_{t} u, L_{t} u\right\rangle \\
& +\left\langle S(\cdot, \alpha(\cdot)) L_{t} u, u\right\rangle+\left\langle S(\cdot, \alpha(\cdot))^{\top} u, L_{t} u\right\rangle+\langle R(\cdot, \alpha(\cdot)) u, u\rangle \\
= & \left\langle\left[\widehat{L}_{t}^{*} G(T, \alpha(T)) \widehat{L}_{t}+L_{t}^{*} Q(\cdot, \alpha(\cdot)) L_{t}+S(\cdot, \alpha(\cdot)) L_{t}+L_{t}^{*} S(\cdot, \alpha(\cdot))^{\top}+R(\cdot, \alpha(\cdot))\right] u, u\right\rangle .
\end{aligned}
$$

Thus we complete the proof.

From the representation of the cost functional, we have the following simple corollary.

Corollary 3.4. Let (H1)-(H2) hold and $t \in[0, T)$ be given. For any $x \in \mathbb{R}^{n}, \epsilon \in \mathbb{R}$ and $u(\cdot), v(\cdot) \in \mathcal{U}[t, T]$, the following holds:

$$
J(t, x, i ; u(\cdot)+\epsilon v(\cdot))=J(t, x, i ; u(\cdot))+\epsilon^{2} J^{0}(t, 0, i ; v(\cdot))+2 \epsilon \mathbb{E} \int_{t}^{T}\langle\bar{M}(t, i)(x, u)(s), v(s)\rangle \mathrm{d} s,
$$

where

$$
\begin{aligned}
\bar{M}(t, i)(x, u)(s):= & B(s, \alpha(s))^{\top} Y^{u}(s ; t, x, i)+D(s, \alpha(s))^{\top} Z^{u}(s ; t, x, i) \\
& +S(s, \alpha(s)) X^{u}(s ; t, x, i)+R(s, \alpha(s)) u(s)+\rho(s, \alpha(s)), \quad s \in[t, T] .
\end{aligned}
$$

Consequently, the map $u(\cdot) \mapsto J(t, x, i ; u(\cdot))$ is Fréchet differentiable with the Fréchet derivative given by

$$
\mathcal{D} J(t, x, i ; u(\cdot))(s)=2 \bar{M}(t, i)(x, u)(s), \quad s \in[t, T],
$$

and (3.12) can also be written as

$$
J(t, x, i ; u(\cdot)+\epsilon v(\cdot))=J(t, x, i ; u(\cdot))+\epsilon^{2} J^{0}(t, 0, i ; v(\cdot))+\epsilon \mathbb{E} \int_{t}^{T}\langle\mathcal{D} J(t, x, i ; u(\cdot))(s), v(s)\rangle \mathrm{d} s .
$$

Proof. From Proposition 3.1, we have

$$
\begin{aligned}
& J(t, x, i ; u(\cdot)+\epsilon v(\cdot)) \\
& =\left\langle M_{2}(t, i)(u+\epsilon v), u+\epsilon v\right\rangle+2\left\langle M_{1}(t, i) x, u+\epsilon v\right\rangle+\left\langle M_{0}(t, i) x, x\right\rangle+2\left\langle\nu_{t}, u+\epsilon v\right\rangle+2\left\langle y_{t}, x\right\rangle+c_{t} \\
& =\left\langle M_{2}(t, i) u, u\right\rangle+2 \epsilon\left\langle M_{2}(t, i) u, v\right\rangle+\epsilon^{2}\left\langle M_{2}(t, i) v, v\right\rangle+2\left\langle M_{1}(t, i) x, u\right\rangle+2 \epsilon\left\langle M_{1}(t, i) x, v\right\rangle+\left\langle M_{0}(t, i) x, x\right\rangle \\
& \quad+2\left\langle\nu_{t}, u\right\rangle+2 \epsilon\left\langle\nu_{t}, v\right\rangle+2\left\langle y_{t}, x\right\rangle+c_{t} \\
& =J(t, x, i ; u(\cdot))+\epsilon^{2} J^{0}(t, 0 ; v(\cdot))+2 \epsilon\left\langle M_{2}(t, i) u+M_{1}(t, i) x+\nu_{t}, v\right\rangle .
\end{aligned}
$$

From the representation of $M_{1}(t, i), M_{2}(t, i)$ and $\nu_{t}$ in Proposition 3.1 and the fact

$$
X^{u}(\cdot ; t, x, i)=X_{0}^{u}(\cdot ; t, x, i)+X^{0}(\cdot ; t, 0, i),
$$

we have

$$
\begin{aligned}
\left(M_{2}(t, i) u\right)(s)+\left(M_{1}(t, i) x\right)(s)+\nu_{t}(s)= & B(s, \alpha(s))^{\top} Y^{u}(s ; t, x, i)+D(s, \alpha(s))^{\top} Z^{u}(s ; t, x, i) \\
& +S(s, \alpha(s)) X^{u}(s ; t, x, i)+R(s, \alpha(s)) u(s)+\rho(s, \alpha(s)) \\
= & \bar{M}(t, i)(x, u)(s), \quad s \in[t, T] .
\end{aligned}
$$




\section{Open-LOOP SOLVABILITIES}

We first present the equivalence between the open-loop solvability and the corresponding forward-backward differential equation system.

Theorem 4.1. Let $(\mathrm{H} 1)-(\mathrm{H} 2)$ hold and $(t, x, i) \in[t, T] \times \mathbb{R}^{n} \times \mathcal{S}$ be given. An element $u(\cdot) \in \mathcal{U}[t, T]$ is an openloop optimal control of Problem (M-SLQ) if and only if $J^{0}(t, 0, i ; v(\cdot)) \geq 0, \forall v(\cdot) \in \mathcal{U}[t, T]$ and the following stationary condition hold:

$$
\begin{aligned}
& B(s, \alpha(s))^{\top} Y^{u}(s ; t, x, i)+D(s, \alpha(s))^{\top} Z^{u}(s ; t, x, i) \\
& +S(s, \alpha(s)) X^{u}(s ; t, x, i)+R(s, \alpha(s)) u(s)+\rho(s, \alpha(s))=0, \quad s \in[t, T],
\end{aligned}
$$

where $\left(X^{u}(\cdot ; t, x, i), Y^{u}(\cdot ; t, x, i), Z^{u}(\cdot ; t, x, i), \Gamma^{u}(\cdot ; t, x, i)\right)$ is an adapted solution to FBSDE (2.8).

Proof. By definition, $u(\cdot)$ is an open-loop optimal control if and only if the following inequality holds:

$$
J(t, x, i ; u(\cdot)+\epsilon v(\cdot))-J(t, x, i ; u) \geqslant 0, \quad \forall v(\cdot) \in \mathcal{U}[t, T] .
$$

From Corollary 3.4, we have

$$
J(t, x, i ; u(\cdot)+\epsilon v(\cdot))-J(t, x, i ; u)=\epsilon^{2} J^{0}(t, 0, i ; v(\cdot))+2 \epsilon \mathbb{E} \int_{t}^{T}\langle\bar{M}(t, i)(x, u)(s), v(s)\rangle \mathrm{d} s .
$$

Therefore, (4.2) holds if and only if $J^{0}(t, 0, i ; v(\cdot)) \geqslant 0, \forall v(\cdot) \in \mathcal{U}[t, T]$ and $\bar{M}(t, i)(x, u)(s)=0, s \in[t, T]$. Note the definition of $\bar{M}$ in (3.13) and so the proof is completed.

Note that if $u(\cdot)$ happens to be an open-loop optimal control of Problem (M-SLQ), then the stationarity condition (4.1) holds. We call (2.8), together with the stationarity condition (4.1), the optimality system for the open-loop optimal control of Problem (M-SLQ).

Next we investigate the relationships between open-loop solvability and uniform convexity of the cost functional. We first introduce the definition of uniform convexity, which is from Zalinescu [33] or [32].

Definition 4.2. For a general normed space $(\mathbb{H},\|\cdot\|)$, the function $f:(\mathbb{H},\|\cdot\|) \mapsto \overline{\mathbb{R}}$ is said to be uniformly convex if there exists $h: \mathbb{R}_{+} \mapsto \overline{\mathbb{R}}_{+}$with $h(t)>0$ for $t>0$ and $h(0)=0$ such that

$$
f(\epsilon x+(1-\epsilon) y) \leqslant \epsilon f(x)+(1-\epsilon) f(y)-\epsilon(1-\epsilon) h(\|x-y\|), \forall x, y \in \operatorname{dom} f, \epsilon \in[0,1] .
$$

Proposition 4.3. The cost functional $J(t, x, i ; u(\cdot))$ is uniformly convex if and only if $M_{2}(t, i) \geqslant \epsilon I$ for some $\epsilon>0$, which is also equivalent to

$$
J^{0}(t, 0, i ; u(\cdot)) \geqslant \epsilon \mathbb{E} \int_{t}^{T}|u(s)|^{2} \mathrm{~d} s, \quad \forall u(\cdot) \in \mathcal{U}[t, T]
$$

for some $\epsilon>0$. 
Proof. From Proposition 3.1, we see that for any $u(\cdot), v(\cdot) \in \mathcal{U}[t, T]$ and $\epsilon \in[0,1]$,

$$
\begin{aligned}
J( & t, x, i ; \epsilon u(\cdot)+(1-\epsilon) v(\cdot)) \\
= & \left\langle M_{2}(t, i)(\epsilon u+(1-\epsilon) v, \epsilon u+(1-\epsilon) v\rangle+2\left\langle M_{1}(t, i) x, \epsilon u+(1-\epsilon) v\right\rangle\right. \\
& +\left\langle M_{0}(t, i) x, x\right\rangle+2\left\langle\nu_{t}, \epsilon u+(1-\epsilon) v\right\rangle+2\left\langle y_{t}, x\right\rangle+c_{t} \\
= & \epsilon\left[\left\langle M_{2}(t, i) u, u\right\rangle+2\left\langle M_{1}(t, i) x, u\right\rangle+\left\langle M_{0}(t, i) x, x\right\rangle+2\left\langle\nu_{t}, u\right\rangle+2\left\langle y_{t}, x\right\rangle+c_{t}\right] \\
& +(1-\epsilon)\left[\left\langle M_{2}(t, i) v, v\right\rangle+2\left\langle M_{1}(t, i) x, v\right\rangle+\left\langle M_{0}(t, i) x, x\right\rangle+2\left\langle\nu_{t}, v\right\rangle+2\left\langle y_{t}, x\right\rangle+c_{t}\right] \\
& -\epsilon(1-\epsilon)\left\langle M_{2}(t, i)(u-v), u-v\right\rangle .
\end{aligned}
$$

Thus, it follows from the definition of uniformly convex that the cost functional $J(t, x, i ; u(\cdot))$ is uniformly convex if and only if there exists $h: \mathbb{R}_{+} \mapsto \overline{\mathbb{R}}_{+}$with $h(t)>0$ for $t>0$ and $h(0)=0$ such that

$$
\left\langle M_{2}(t, i)(u-v), u-v\right\rangle \geqslant h(\|u-v\|)
$$

which is equivalent to $M_{2}(t, i) \geqslant \epsilon I$ for some $\epsilon>0$. From Proposition 3.1, we have

$$
J^{0}(t, 0, i ; u(\cdot))=\left\langle M_{2}(t, i) u, u\right\rangle .
$$

Therefore, $M_{2}(t, i)>\epsilon I$ for some $\epsilon>0$ if and only if

$$
J^{0}(t, 0, i ; u(\cdot)) \geqslant \epsilon \mathbb{E} \int_{t}^{T}|u(s)|^{2} \mathrm{~d} s, \quad \forall u(\cdot) \in \mathcal{U}[t, T]
$$

Thus the proof is completed.

Remark 4.4. From the definition of uniform convexity, one can easily verify that $J^{0}(t, x, i ; u(\cdot))$ is uniformly convex if and only if (4.3) is satisfied. Therefore, the uniform convexity of $J(t, x, i ; u(\cdot))$ is equivalent to the uniform convexity of $J^{0}(t, x, i ; u(\cdot))$.

It is obvious that if the following standard conditions

$$
G(T, i) \geqslant 0, \quad R(s, i) \geqslant \delta I, \quad Q(s, i)-S(s, i)^{\top} R(s, i)^{-1} S(s, i) \geqslant 0, \quad i \in \mathcal{S}, \quad \text { a.e. } \quad s \in[0, T]
$$

hold for some $\delta>0$, then

$$
\begin{aligned}
M_{2}(t, i)= & \widehat{L}_{t}^{*} G(T, \alpha(T)) \widehat{L}_{t}+L_{t}^{*}\left[Q(\cdot, \alpha(\cdot))-S(\cdot, \alpha(\cdot))^{\top} R(\cdot, \alpha(\cdot))^{-1} S(\cdot, \alpha(\cdot))\right] L_{t} \\
& +\left[L_{t}^{*} S(\cdot, \alpha(\cdot))^{\top} R(\cdot, \alpha(\cdot))^{-\frac{1}{2}}+R(\cdot, \alpha(\cdot))^{\frac{1}{2}}\right]\left[R(\cdot, \alpha(\cdot))^{-\frac{1}{2}} S\left(\cdot, \alpha(\cdot) L_{t}+R(\cdot, \alpha(\cdot))^{\frac{1}{2}}\right]\right. \\
\geqslant & 0
\end{aligned}
$$

which means that the functional $u(\cdot) \mapsto J^{0}(t, 0, i ; u(\cdot))$ is convex. In fact, one actually has the uniform convexity of the cost functional $J^{0}(t, 0, i ; u(\cdot))$ under standard conditions (4.4). We first present a lemma for proving the uniform convexity of $J^{0}(t, x, i ; u(\cdot))$.

Lemma 4.5. For any $u(\cdot) \in \mathcal{U}[t, T]$, let $X_{0}^{u}(\cdot ; t, 0, i)$ be the solution of $(2.8)$ with $x=0, b(\cdot)=\sigma(\cdot)=0$. Then for any $\Theta(\cdot, i) \in L^{2}\left(t, T ; \mathbb{R}^{m \times n}\right), i \in \mathcal{S}$, there exists a constant $\gamma>0$ such that

$$
\mathbb{E} \int_{t}^{T}\left|u(s)-\Theta(s, \alpha(s)) X_{0}^{u}(s ; t, 0, i)\right|^{2} \mathrm{~d} s \geqslant \gamma \mathbb{E} \int_{t}^{T}|u(s)|^{2} \mathrm{~d} s, \quad \forall u(\cdot) \in \mathcal{U}[t, T] .
$$


Proof. The proof is similar to Lemma 2.3 of Sun et al. [20] and so we omit it here.

Proposition 4.6. Let (H1)-(H2) and (4.4) hold. Then for any $(t, i) \in[0, T) \times \mathcal{S}$, the map $u(\cdot) \mapsto J^{0}(t, 0, i ; u(\cdot))$ is uniformly convex.

Proof. By Lemma 4.5 (taking $\Theta(\cdot, \alpha(\cdot))=-R(\cdot, \alpha(\cdot))^{-1} S(\cdot, \alpha(\cdot))$ ), we have

$$
\begin{aligned}
& J^{0}(t, 0, i ; u(\cdot))=\mathbb{E}\{\langle\left.G(T, \alpha(T)) X_{0}^{u}(T ; t, 0, i), X_{0}^{u}(T ; t, 0, i)\right\rangle \\
&+\int_{t}^{T}\left[\left\langle Q(s, \alpha(s)) X_{0}^{u}(s ; t, 0, i), X_{0}^{u}(s ; t, 0, i)\right\rangle\right. \\
&\left.\left.+2\left\langle S(s, \alpha(s)) X_{0}^{u}(s ; t, 0, i), u(s)\right\rangle+\langle R(s, \alpha(s)) u(s), u(s)\rangle\right] \mathrm{d} s\right\} \\
& \geqslant \mathbb{E} \int_{t}^{T}\left[\left\langle Q(s, \alpha(s)) X_{0}^{u}(s ; t, 0, i), X_{0}^{u}(s ; t, 0, i)\right\rangle\right. \\
&\left.+2\left\langle S(s, \alpha(s)) X_{0}^{u}(s ; t, 0, i), u(s)\right\rangle+\langle R(s, \alpha(s)) u(s), u(s)\rangle\right] \mathrm{d} s \\
&=\mathbb{E} \int_{t}^{T}\left[\left\langle\left[Q(s, \alpha(s))-S(s, \alpha(s))^{\top} R(s, \alpha(s))^{-1} S(s, \alpha(s))\right] X_{0}^{u}(s ; t, 0, i), X_{0}^{u}(s ; t, 0, i)\right\rangle\right. \\
& \quad+\left\langle R(s, \alpha(s))\left[u(s)+R(s, \alpha(s))^{-1} S(s, \alpha(s)) X_{0}^{u}(s ; t, 0, i)\right],\right. \\
&\left.\left.\quad u(s)+R(s, \alpha(s))^{-1} S(s, \alpha(s)) X_{0}^{u}(s ; t, 0, i)\right\rangle\right] \mathrm{d} s \\
& \geqslant \delta \mathbb{E} \int_{t}^{T}\left|u(s)+R(s, \alpha(s))^{-1} S(s, \alpha(s)) X_{0}^{u}(s ; t, 0, i)\right|^{2} \mathrm{~d} s \\
& \geqslant \delta \gamma \mathbb{E} \int_{t}^{T}|u(s)|^{2} \mathrm{~d} s, \quad \forall u(\cdot) \in \mathcal{U}[t, T],
\end{aligned}
$$

for some $\gamma>0$. This completes the proof.

Next, we show that the uniform convexity of $J^{0}(t, x, i ; u(\cdot))$ implies the open-loop solvability of Problem (M-SLQ).

Theorem 4.7. Let (H1)-(H2) hold. Suppose the map $u(\cdot) \mapsto J^{0}(t, 0, i ; u(\cdot))$ is uniformly convex. Then Problem (M-SLQ) is uniquely open-loop solvable, and there exists a constant $\gamma \in \mathbb{R}$ such that

$$
V^{0}(t, x, i) \geqslant \gamma|x|^{2}, \quad \forall(t, x, i) \in[0, T] \times \mathbb{R}^{n} \times \mathcal{S} .
$$

Note that in the above, the constant $\gamma$ does not have to be nonnegative.

Proof. First of all, by the uniform convexity of $u(\cdot) \mapsto J^{0}(t, 0, i ; u(\cdot))$, we may assume that

$$
J^{0}(t, 0, i ; u(\cdot)) \geqslant \lambda \mathbb{E} \int_{t}^{T}|u(s)|^{2} \mathrm{~d} s, \quad \forall u(\cdot) \in \mathcal{U}[0, T]
$$

for some $\lambda>0$. Thus, $u(\cdot) \mapsto J^{0}(t, x, i ; u(\cdot))$ is uniformly convex given $(t, x, i) \in[0, T) \times \mathbb{R}^{n} \times \mathcal{S}$. By Corollary 3.4 , we have

$$
J(t, x, i ; u(\cdot))=J(t, x, i ; 0)+J^{0}(t, 0, i ; u(\cdot))+\mathbb{E} \int_{t}^{T}\langle\mathcal{D} J(t, x, i ; 0)(s), u(s)\rangle \mathrm{d} s
$$




$$
\begin{aligned}
& \geqslant J(t, x, i ; 0)+J^{0}(t, 0, i ; u(\cdot))-\frac{\lambda}{2} \mathbb{E} \int_{t}^{T}|u(s)|^{2} \mathrm{~d} s-\frac{1}{2 \lambda} \mathbb{E} \int_{t}^{T}|\mathcal{D} J(t, x, i ; 0)(s)|^{2} \mathrm{~d} s \\
& \geqslant \frac{\lambda}{2} \mathbb{E} \int_{t}^{T}|u(s)|^{2} \mathrm{~d} s+J(t, x, i ; 0)-\frac{1}{2 \lambda} \mathbb{E} \int_{t}^{T}|\mathcal{D} J(t, x, i ; 0)(s)|^{2} \mathrm{~d} s, \quad \forall u(\cdot) \in \mathcal{U}[t, T] .
\end{aligned}
$$

Consequently, by a standard argument involving minimizing sequence and locally weak compactness of Hilbert spaces, we see that for any given initial pair $(t, x, i) \in[0, T) \times \mathbb{R}^{n} \times \mathcal{S}$, Problem (M-SLQ) admits a unique open-loop optimal control. Moreover, when $b(\cdot), \sigma(\cdot), g(\cdot, \cdot), q(\cdot, \cdot), \rho(\cdot, \cdot)=0,(4.7)$ implies that

$$
V^{0}(t, x, i) \geqslant J^{0}(t, x, i ; 0)-\frac{1}{2 \lambda} \mathbb{E} \int_{t}^{T}\left|\mathcal{D} J^{0}(t, x, i ; 0)(s)\right|^{2} \mathrm{~d} s
$$

Note that the functions on the right-hand side of (4.8) are quadratic in $x$ and continuous in $t$, (4.6) follows immediately.

\section{Closed-Loop solvabilities}

In this section, we establish the equivalence between the closed-loop solvability and the existence of a regular solution to Riccati equation. In the following, we first introduce some notation and Riccati equation. Let

$$
\begin{aligned}
& \widehat{S}(s, i):=B(s, i)^{\top} P(s, i)+D(s, i)^{\top} P(s, i) C(s, i)+S(s, i), \\
& \widehat{R}(s, i):=R(s, i)+D(s, i)^{\top} P(s, i) D(s, i) .
\end{aligned}
$$

The Riccati equation associated with Problem (M-SLQ) is

$$
\left\{\begin{aligned}
\dot{P}(s, i) & +P(s, i) A(s, i)+A(s, i)^{\top} P(s, i)+C(s, i)^{\top} P(s, i) C(s, i) \\
& -\widehat{S}(s, i)^{\top} \widehat{R}(s, i)^{\dagger} \widehat{S}(s, i)+Q(s, i)+\sum_{k=1}^{D} \lambda_{i k}(s) P(s, k)=0, \quad \text { a.e. } s \in[t, T], \\
P(T, i) & =G(T, i), \quad i \in \mathcal{S} .
\end{aligned}\right.
$$

Definition 5.1. A solution $P(\cdot, \cdot) \in C\left([t, T] \times \mathcal{S} ; \mathbb{S}^{n}\right)$ of $(5.2)$ is said to be regular if for any $i \in \mathcal{S}$

$$
\begin{aligned}
\mathcal{R}(\widehat{S}(s, i)) & \subseteq \mathcal{R}(\widehat{R}(s, i)), \quad \text { a.e. } \quad s \in[t, T], \\
\widehat{R}(\cdot, i)^{\dagger} \widehat{S}(\cdot, i) & \in L^{2}\left(t, T ; \mathbb{R}^{m \times n}\right), \\
\widehat{R}(s, i) & \geqslant 0, \quad \text { a.e. } \quad s \in[t, T] .
\end{aligned}
$$

A solution $P(\cdot, \cdot)$ of $(5.2)$ is said to be strongly regular if for any $i \in \mathcal{S}$

$$
\widehat{R}(s, i) \geqslant \lambda I, \quad \text { a.e. } s \in[t, T],
$$

for some $\lambda>0$. The Riccati equation (5.2) is said to be (strongly) regularly solvable, if it admits a (strongly) regular solution.

Clearly, condition (5.4) implies (5.3). Thus, a strongly regular solution $P(\cdot, \cdot)$ must be regular. Moreover, if a regular solution of (5.2) exists, it must be unique. 
Theorem 5.2. Let (H1)-(H2) hold. Problem (M-SLQ) is closed-loop solvable if and only if the Riccati equation (5.2) admits a regular solution $P(\cdot, \cdot) \in C\left([t, T] \times \mathcal{S} ; \mathbb{S}^{n}\right)$ and the solution $(\eta(\cdot), \zeta(\cdot), \xi(\cdot))$ of the following BSDE:

$$
\left\{\begin{aligned}
\mathrm{d} \eta(s)=-\{[ & \left.A(s, \alpha(s))^{\top}-\widehat{S}(s, \alpha(s))^{\top} \widehat{R}(s, \alpha(s))^{\dagger} B(s, \alpha(s))^{\top}\right] \eta(s) \\
& +\left[C(s, \alpha(s))^{\top}-\widehat{S}(s, \alpha(s))^{\top} \widehat{R}(s, \alpha(s))^{\dagger} D(s, \alpha(s))^{\top}\right] \zeta(s) \\
& +\left[C(s, \alpha(s))^{\top}-\widehat{S}(s, \alpha(s))^{\top} \widehat{R}(s, \alpha(s))^{\dagger} D(s, \alpha(s))^{\top}\right] P(s, \alpha(s)) \sigma(s) \\
& \left.-\widehat{S}(s, \alpha(s))^{\top} \widehat{R}(s, \alpha(s))^{\dagger} \rho(s, \alpha(s))+P(s, \alpha(s)) b(s)+q(s, \alpha(s))\right\} \mathrm{d} s \\
& +\zeta(s) \mathrm{d} W(s)+\sum_{l, k=1}^{D} \xi_{l k}(s) d \widetilde{N}_{l k}(s), \quad s \in[t, T], \\
\eta(T)=g(T, & \alpha(T)),
\end{aligned}\right.
$$

satisfies

$$
\left\{\begin{array}{l}
\widehat{\rho}(s) \in \mathcal{R}(\widehat{R}(s, \alpha(s))), \quad \text { a.e. a.s. } \\
\widehat{R}(s, \alpha(s))^{\dagger} \widehat{\rho}(s) \in L_{\mathbb{F}}^{2}\left(0, T ; \mathbb{R}^{m}\right)
\end{array}\right.
$$

with

$$
\widehat{\rho}(s)=B(s, \alpha(s))^{\top} \eta(s)+D(s, \alpha(s))^{\top} \zeta(s)+D(s, \alpha(s))^{\top} P(s, \alpha(s)) \sigma(s)+\rho(s, \alpha(s)) .
$$

In this case, Problem (M-SLQ) is closed-loop solvable, and the closed-loop optimal strategy $\left(\Theta^{*}(\cdot, \alpha(\cdot)), v^{*}(\cdot)\right)$ admits the following representation:

$$
\left\{\begin{aligned}
\Theta^{*}(s, \alpha(s)) & =-\widehat{R}(s, \alpha(s))^{\dagger} \widehat{S}(s, \alpha(s))+\left[I-\widehat{R}(s, \alpha(s))^{\dagger} \widehat{R}(s, \alpha(s))\right] \Pi(s, \alpha(s)), \\
v^{*}(s) & =-\widehat{R}(s, \alpha(s))^{\dagger} \widehat{\rho}(s)+\left[I-\widehat{R}(s, \alpha(s))^{\dagger} \widehat{R}(s, \alpha(s))\right] \nu(s),
\end{aligned}\right.
$$

for some $\Pi(\cdot, i) \in L^{2}\left(t, T ; \mathbb{R}^{m \times n}\right)$ and $\nu(\cdot) \in L_{\mathbb{F}}^{2}\left(t, T ; \mathbb{R}^{m}\right)$, and the value function is given by

$$
V(t, x, i)=\mathbb{E}\left\{\langle P(t, i) x, x\rangle+2\langle\eta(t), x\rangle+\int_{t}^{T}\left[\widehat{P}(s)-\left\langle\widehat{R}(s, \alpha(s))^{\dagger} \widehat{\rho}(s), \widehat{\rho}(s)\right\rangle\right] \mathrm{d} s\right\}
$$

where $\widehat{P}(s):=\langle P(s, \alpha(s)) \sigma(s)+2 \zeta(s), \sigma(s)\rangle+2\langle\eta(s), b(s)\rangle$.

Proof. Necessity. Let $\left(\Theta^{*}(\cdot, \alpha(\cdot)), v^{*}(\cdot)\right)$ be a closed-loop optimal strategy of Problem (M-SLQ) and set

$$
\left(X^{*}(\cdot), Y^{*}(\cdot), Z^{*}(\cdot), \Gamma^{*}(\cdot)\right):=\left(X^{\Theta^{*}, v^{*}}(\cdot ; t, x, i), Y^{\Theta^{*}, v^{*}}(\cdot ; t, x, i), Z^{\Theta^{*}, v^{*}}(\cdot ; t, x, i), \Gamma^{\Theta^{*}, v^{*}}(\cdot ; t, x, i)\right) .
$$

Then the following stationary condition holds:

$$
\begin{aligned}
B(s, \alpha(s))^{\top} Y^{*}(s) & +D(s, \alpha(s))^{\top} Z^{*}(s)+\left[S(s, \alpha(s))+R(s, \alpha(s)) \Theta^{*}(s, \alpha(s))\right] X^{*}(s) \\
& +R(s, \alpha(s)) v^{*}(s)+\rho(s, \alpha(s))=0 \quad \text { a.e. a.s. }
\end{aligned}
$$

Since the above admits a solution for each $(x, i) \in \mathbb{R}^{n} \times \mathcal{S}$, and $\left(\Theta^{*}(\cdot, \alpha(\cdot)), v^{*}(\cdot)\right)$ is independent of $(x, i)$, by subtracting soulutions corresponding to $(x, i)$ and $(0, i)$, the later from the former, we see that for any 
$(x, i) \in \mathbb{R}^{n} \times \mathcal{S}$, as long as $(X(\cdot ; t, x, i), Y(\cdot ; t, x, i), Z(\cdot ; t, x, i), \Gamma(\cdot ; t, x, i))$ is an adapted solution to the following FBSDE

$$
\left\{\begin{aligned}
\mathrm{d} X(s ; t, x, i)= & {\left[A(s, \alpha(s))+B(s, \alpha(s)) \Theta^{*}(s, \alpha(s))\right] X(s ; t, x, i) \mathrm{d} s } \\
& +\left[C(s, \alpha(s))+D(s, \alpha(s)) \Theta^{*}(s, \alpha(s))\right] X(s ; t, x, i) \mathrm{d} W(s), \\
\mathrm{d} Y(s ; t, x, i)= & -\left[A(s, \alpha(s))^{\top} Y(s ; t, x, i)+C(s, \alpha(s))^{\top} Z(s ; t, x, i)\right. \\
& \left.+\left[Q(s, \alpha(s))+S(s, \alpha(s))^{\top} \Theta^{*}(s, \alpha(s))\right] X(s ; t, x, i)\right] \mathrm{d} s \\
& +Z(s ; t, x, i) \mathrm{d} W(s)+\sum_{l, k=1}^{D} \Gamma_{l k}(s ; t, x, i) \mathrm{d} \widetilde{N}_{l k}(s), \quad s \in[t, T] \\
X(t ; t, x, i)= & x, \quad \alpha(t)=i, \quad Y(T ; t, x, i)=G(T, \alpha(T)) X(T ; t, x, i)
\end{aligned}\right.
$$

one can have the following stationary condition:

$$
\begin{aligned}
B(s, \alpha(s))^{\top} Y(s ; t, x, i) & +D(s, \alpha(s))^{\top} Z(s ; t, x, i) \\
& +\left[S(s, \alpha(s))+R(s, \alpha(s)) \Theta^{*}(s, \alpha(s))\right] X(s ; t, x, i)=0, \quad \text { a.e. a.s.. }
\end{aligned}
$$

Clearly,

$$
\begin{aligned}
& (X(\cdot ; t, x, i), Y(\cdot ; t, x, i), Z(\cdot ; t, x, i), \Gamma(\cdot ; t, x, i)) \\
& =\left(X_{0}^{\Theta^{*}, 0}(\cdot ; t, x, i), Y_{0}^{\Theta^{*}, 0}(\cdot ; t, x, i), Z_{0}^{\Theta^{*}, 0}(\cdot ; t, x, i), \Gamma_{0}^{\Theta^{*}, 0}(\cdot ; t, x, i)\right) .
\end{aligned}
$$

Let $e_{i}$ denote the unit vector of $\mathbb{R}^{n}$ whose $i$-th component is one. Define, for $t \leq s \leq T$,

$$
\begin{aligned}
X(s ; t, i) & :=\left(X\left(s ; t, e_{1}, i\right), \ldots, X\left(s ; t, e_{n}, i\right)\right), \quad Y(s ; t, i):=\left(Y\left(s ; t, e_{1}, i\right), \ldots, Y\left(s ; t, e_{n}, i\right)\right), \\
Z(s ; t, i) & :=\left(Z\left(s ; t, e_{1}, i\right), \ldots, Z\left(s ; t, e_{n}, i\right)\right), \quad \Gamma_{k}(s ; t, i):=\left(\Gamma_{k}\left(s ; t, e_{1}, i\right), \ldots, \Gamma_{k}\left(s ; t, e_{n}, i\right)\right) .
\end{aligned}
$$

It is easy to verify that, for $t \leq s \leq T$

$$
\begin{aligned}
& X(s ; t, x, i)=X(s ; t, i) x, \quad Y(s ; t, x, i)=Y(s ; t, i) x \\
& Z(s ; t, x, i)=Z(s ; t, i) x, \quad \Gamma_{k}(s ; t, x, i)=\Gamma_{k}(s ; t, i) x .
\end{aligned}
$$

In particular, if we set $P(t, i):=Y(t ; t, i)$, then

$$
Y(t ; t, x, i)=Y(t ; t, i) x=P(t, i) x .
$$

Therefore,

$$
\begin{aligned}
Y(s ; t, i) x & =Y(s ; t, x, i)=Y(s ; s, X(s ; t, x, i), \alpha(s))=Y(s ; s, \alpha(s)) X(s ; t, x, i) \\
& =P(s, \alpha(s)) X(s ; t, i) x, \quad \text { for any } x \in \mathbb{R}^{n}
\end{aligned}
$$

which leads to

$$
Y(s ; t, i)=P(s, \alpha(s)) X(s ; t, i) .
$$


Note that $X(s ; t, i)^{-1}$ exists, and so

$$
P(s, \alpha(s))=Y(s ; t, i) X(s ; t, i)^{-1}
$$

Thus the differentiability of $P(\cdot, \cdot)$ follows from the differentiability of $Y(\cdot ; t, i)$ and $X(\cdot ; t, i)$ in the above. Let $\dot{P}(s, i)$ be the partial derivative of $P$ with respect to $s$. Applying Itô's formula to $P(\cdot, \alpha(\cdot)) X(\cdot ; t, i)$ yields

$$
\begin{aligned}
d[P(s, \alpha(s)) X(s ; t, i)]= & {\left[\dot{P}(s, \alpha(s))+P(s, \alpha(s))\left[A(s, \alpha(s))+B(s, \alpha(s)) \Theta^{*}(s, \alpha(s))\right]\right.} \\
& \left.+\sum_{k=1}^{D} \lambda_{\alpha(s-) k}(s)[P(s, k)-P(s, \alpha(s-))]\right] X(s ; t, i) \mathrm{d} s \\
& +P(s, \alpha(s))\left[C(s, \alpha(s))+D(s, \alpha(s)) \Theta^{*}(s, \alpha(s))\right] X(s ; t, i) \mathrm{d} W(s) \\
& +\sum_{l, k=1}^{D}[P(s, k)-P(s, l)] X(s ; t, i) d \widetilde{N}_{l k}(s) .
\end{aligned}
$$

Also, we observe that $Y(s ; t, i)$ satisfies the following BSDE

$$
\left\{\begin{aligned}
\mathrm{d} Y(s ; t, i)=- & {\left[A(s, \alpha(s))^{\top} Y(s ; t, i)+C(s, \alpha(s))^{\top} Z(s ; t, i)\right.} \\
& \left.+\left[Q(s, \alpha(s))+S(s, \alpha(s)) \Theta^{*}(s, \alpha(s))\right] X(s ; t, i)\right] \mathrm{d} s \\
& +Z(s ; t, i) \mathrm{d} W(s)+\sum_{l, k=1}^{D} \Gamma_{l k}(s ; t, i) \mathrm{d} \widetilde{N}_{l k}(s), \quad s \in[t, T], \\
Y(T ; t, i)=G( & T, \alpha(T)) X(T ; t, i) .
\end{aligned}\right.
$$

Comparing the coefficients of (5.16) and (5.17), we have

$$
\begin{aligned}
Z(s ; t, i) & =P(s, \alpha(s))\left[C(s, \alpha(s))+D(s, \alpha(s)) \Theta^{*}(s, \alpha(s))\right] X(s ; t, i), \\
\Gamma_{k}(s ; t, i) & =[P(s, k)-P(s, \alpha(s-))] X(s ; t, i),
\end{aligned}
$$

and

$$
\begin{aligned}
& \left\{\dot{P}(s, \alpha(s))+A(s, \alpha(s))^{\top} P(s, \alpha(s))+P(s, \alpha(s)) A(s, \alpha(s))+C(s, \alpha(s))^{\top} P(s, \alpha(s)) C(s, \alpha(s))\right. \\
& \quad+\left[P(s, \alpha(s)) B(s, \alpha(s))+C(s, \alpha(s))^{\top} P(s, \alpha(s)) D(s, \alpha(s))+S(s, \alpha(s))^{\top}\right] \Theta^{*}(s, \alpha(s)) \\
& \left.\quad+Q(s, \alpha(s))+\sum_{k=1}^{D} \lambda_{\alpha(s-) k}(s)[P(s, k)-P(s, \alpha(s-))]\right\} X(s ; t, i)=0 .
\end{aligned}
$$


Observing that the inverse of $X(s ; t, i)$ exists, therefore the last equation reduces to

$$
\begin{aligned}
& \dot{P}(s, \alpha(s))+A(s, \alpha(s))^{\top} P(s, \alpha(s))+P(s, \alpha(s)) A(s, \alpha(s))+C(s, \alpha(s))^{\top} P(s, \alpha(s)) C(s, \alpha(s)) \\
& \quad+\left[P(s, \alpha(s)) B(s, \alpha(s))+C(s, \alpha(s))^{\top} P(s, \alpha(s)) D(s, \alpha(s))+S(s, \alpha(s))^{\top}\right] \Theta^{*}(s, \alpha(s)) \\
& \quad+Q(s, \alpha(s))+\sum_{k=1}^{D} \lambda_{\alpha(s-) k}(s)[P(s, k)-P(s, \alpha(s-))]=0 .
\end{aligned}
$$

Now, if we replace $\alpha(s-)$ by $\alpha(s)$ in the above equation, it is still satisfied except countable points of $[t, T]$ due to the right continuous with left limit property of the path of Markov chain $\alpha$. That is,

$$
\begin{aligned}
& \dot{P}(s, \alpha(s))+A(s, \alpha(s))^{\top} P(s, \alpha(s))+P(s, \alpha(s)) A(s, \alpha(s))+C(s, \alpha(s))^{\top} P(s, \alpha(s)) C(s, \alpha(s)) \\
& \quad+\left[P(s, \alpha(s)) B(s, \alpha(s))+C(s, \alpha(s))^{\top} P(s, \alpha(s)) D(s, \alpha(s))+S(s, \alpha(s))^{\top}\right] \Theta^{*}(s, \alpha(s)) \\
& \quad+Q(s, \alpha(s))+\sum_{k=1}^{D} \lambda_{\alpha(s) k}(s)[P(s, k)-P(s, \alpha(s))]=0, \quad \text { a.e. } \quad s \in[t, T] .
\end{aligned}
$$

Observing $\sum_{k=1}^{D} \lambda_{i k}(s)=0$, we can also rewrite the above equation into the following form

$$
\begin{aligned}
\sum_{i=1}^{D}\{\dot{P}( & s, i)+A(s, i)^{\top} P(s, i)+P(s, i) A(s, i)+C(s, i)^{\top} P(s, i) C(s, i) \\
+ & {\left[P(s, i) B(s, i)+C(s, i)^{\top} P(s, i) D(s, i)+S(s, i)^{\top}\right] \Theta^{*}(s, i) } \\
+ & \left.Q(s, i)+\sum_{k=1}^{D} \lambda_{i k}(s) P(s, k)\right\} 1_{\alpha(s)=i}=0, \quad \text { a.e. } \quad s \in[t, T] .
\end{aligned}
$$

Therefore, by taking $\alpha(t)$ for all initial $(t, i)$, we have

$$
\begin{aligned}
& \dot{P}(s, i)+A(s, i)^{\top} P(s, i)+P(s, i) A(s, i)+C(s, i)^{\top} P(s, i) C(s, i) \\
& \quad+\left[P(s, i) B(s, i)+C(s, i)^{\top} P(s, i) D(s, i)+S(s, i)^{\top}\right] \Theta^{*}(s, i) \\
& \quad+Q(s, i)+\sum_{k=1}^{D} \lambda_{i k}(s) P(s, k)=0, i \in \mathcal{S}, \text { a.e. } s \in[t, T] .
\end{aligned}
$$

It follows from (5.14), (5.15) and (5.18), and the definitions of $\widehat{S}(\cdot, \cdot)$ and $\widehat{R}(\cdot, \cdot)$ in $(5.1)$ that the stationary condition (5.12) can be rewritten as

$$
\left[\widehat{S}(s, \alpha(s))+\widehat{R}(s, \alpha(s)) \Theta^{*}(s, \alpha(s))\right] X(s ; t, i)=0, \quad \text { a.e. a.s. }
$$

which yields

$$
\widehat{S}(s, \alpha(s))+\widehat{R}(s, \alpha(s)) \Theta^{*}(s, \alpha(s))=0, \quad \text { a.e. a.s.. }
$$


Thus, from the irreducibility of the Markov chain, we have

$$
\widehat{S}(s, i)+\widehat{R}(s, i) \Theta^{*}(s, i)=0, \forall i \in \mathcal{S}, \quad \text { a.e. },
$$

which implies

$$
\mathcal{R}(\widehat{S}(s, i) \subseteq \mathcal{R}(\widehat{R}(s, i)), \quad \text { a.e. } s \in[t, T]
$$

Using (5.23), we can rewrite (5.22) as

$$
\begin{aligned}
& \dot{P}(s, i)+\left[A(s, i)+B(s, i) \Theta^{*}(s, i)\right]^{\top} P(s, i)+P(s, i)\left[A(s, i)+B(s, i) \Theta^{*}(s, i)\right] \\
& +\left[C(s, i)+D(s, i) \Theta^{*}(s, i)\right]^{\top} P(s, i)\left[C(s, i)+D(s, i) \Theta^{*}(s, i)\right]+\Theta^{*}(s, i)^{\top} R(s, i) \Theta^{*}(s, i) \\
& +S(s, i)^{\top} \Theta^{*}(s, i)+\Theta^{*}(s, i)^{\top} S(s, i)+Q(s, i)+\sum_{k=1}^{D} \lambda_{i k}(s) P(s, k)=0, \quad \text { a.e. } s \in[t, T] .
\end{aligned}
$$

Since $P(T, i)=G(T, i) \in \mathbb{S}^{n}$ and $Q(\cdot, \cdot), R(\cdot, \cdot)$ are symmetric, we must have $P(\cdot, \cdot) \in C\left([t, T] \times S ; \mathbb{S}^{n}\right)$ due to the uniqueness of the solution of $(5.24)$. Let $\widehat{R}(\cdot, \cdot)^{\dagger}$ be the pseudo inverse of $\widehat{R}(\cdot, \cdot)$, then the solution of $(5.23)$ admits the following representation

$$
\Theta^{*}(s, i)=-\widehat{R}(s, i)^{\dagger} \widehat{S}(s, i)+\left(I-\widehat{R}(s, i)^{\dagger} \widehat{R}(s, i)\right) \Pi(s, i),
$$

for some $\Pi(\cdot, i) \in L^{2}\left(t, T ; \mathbb{R}^{m \times n}\right)$. Thus, making use of (5.23), we have

$$
\begin{aligned}
& \widehat{S}(s, i)^{\top} \Theta^{*}(s, i) \\
& =-\Theta^{*}(s, i)^{\top} \widehat{R}(s, i) \Theta^{*}(s, i) \\
& =-\Theta^{*}(s, i)^{\top} \widehat{R}(s, i)\left[-\widehat{R}(s, i)^{\dagger} \widehat{S}(s, i)+\left(I-\widehat{R}(s, i)^{\dagger} \widehat{R}(s, i)\right) \Pi(s, i)\right] \\
& =-\widehat{S}(s, i)^{\top} \widehat{R}(s, i)^{\dagger} \widehat{S}(s, i) .
\end{aligned}
$$

Substituting the above equation into (5.22) we obtain

$$
\begin{aligned}
\dot{P}(s, i) & +A(s, i)^{\top} P(s, i)+P(s, i) A(s, i)+C(s, i)^{\top} P(s, i) C(s, i) \\
& -\widehat{S}(s, i)^{\top} \widehat{R}(s, i)^{\dagger} \widehat{S}(s, i)+Q(s, i)+\sum_{k=1}^{D} \lambda_{i k}(s) P(s, k)=0, \quad \text { a.e. } \quad s \in[t, T] .
\end{aligned}
$$

Therefore, Riccati equation (5.2) follows.

Next, we figure out $v^{*}(\cdot)$. For $s \in[t, T]$, let

$$
\left\{\begin{aligned}
\eta(s)=Y^{*}(s) & -P(s, \alpha(s)) X^{*}(s) \\
\zeta(s)=Z^{*}(s) & -P(s, \alpha(s))\left[C(s, \alpha(s))+D(s, \alpha(s)) \Theta^{*}(s, \alpha(s))\right] X^{*}(s) \\
& -P(s, \alpha(s)) D(s, \alpha(s)) v^{*}(s)-P(s, \alpha(s)) \sigma(s) \\
\xi_{l k}(s)=\Gamma_{l k}^{*}(s) & -[P(s, k)-P(s, l)] X^{*}(s) .
\end{aligned}\right.
$$

Then

$$
\mathrm{d} \eta(s)=\mathrm{d} Y^{*}(s)-d P(s, \alpha(s)) \cdot X^{*}(s)-P(s, \alpha(s)) \mathrm{d} X^{*}(s)
$$




$$
\begin{aligned}
& =-\left[A(s, \alpha(s))^{\top} Y^{*}(s)+C(s, \alpha(s))^{\top} Z^{*}(s)+\left(Q(s, \alpha(s))+S(s, \alpha(s))^{\top} \Theta^{*}(s, \alpha(s))\right) X^{*}(s)\right. \\
& \left.+S(s, \alpha(s))^{\top} v^{*}(s)+q(s, \alpha(s))\right] \mathrm{d} s+Z^{*}(s) \mathrm{d} W(s)+\sum_{l, k=1}^{D} \Gamma_{l k}^{*}(s) \mathrm{d} \widetilde{N}_{l k}(s) \\
& +\left\{\left[A(s, \alpha(s))^{\top} P(s, \alpha(s))+P(s, \alpha(s)) A(s, \alpha(s))+C(s, \alpha(s))^{\top} P(s, \alpha(s)) C(s, \alpha(s))\right.\right. \\
& \left.-\widehat{S}(s, \alpha(s))^{\top} \widehat{R}(s, \alpha(s))^{\dagger} \widehat{S}(s, \alpha(s))+Q(s, \alpha(s))\right] X^{*}(s) \\
& -P(s, \alpha(s))\left[\left(A(s, \alpha(s))+B(s, \alpha(s)) \Theta^{*}(s, \alpha(s))\right) X^{*}(s)\right. \\
& \left.\left.+B(s, \alpha(s)) v^{*}(s)+b(s)\right]\right\} \mathrm{d} s \\
& -P(s, \alpha(s))\left[\left(C(s, \alpha(s))+D(s, \alpha(s)) \Theta^{*}(s, \alpha(s))\right) X^{*}(s)+D(s, \alpha(s)) v^{*}(s)\right. \\
& +\sigma(s)] \mathrm{d} W(s)-\sum_{l, k=1}^{D}[P(s, k)-P(s, l)] X^{*}(s) d \widetilde{N}_{l k}(s) \\
& =-\left[A(s, \alpha(s))^{\top} \eta(s)+C(s, \alpha(s))^{\top} \zeta(s)+\widehat{S}(s, \alpha(s))^{\top}\left[\Theta^{*}(s, \alpha(s)) X^{*}(s)+v^{*}(s)\right]\right. \\
& +C(s, \alpha(s))^{\top} P(s, \alpha(s)) \sigma(s)+P(s, \alpha(s)) b(s)+q(s, \alpha(s)) \\
& \left.+\widehat{S}(s, \alpha(s))^{\top} \widehat{R}(s, \alpha(s))^{\dagger} \widehat{S}(s, \alpha(s)) X^{*}(s)\right] \mathrm{d} s+\zeta(s) \mathrm{d} W(s)+\sum_{l, k=1}^{D} \xi_{l k}(s) \widetilde{N}_{l k}(s) \\
& =-\left[A(s, \alpha(s))^{\top} \eta(s)+C(s, \alpha(s))^{\top} \zeta(s)+\widehat{S}(s, \alpha(s))^{\top} v^{*}(s)+C(s, \alpha(s))^{\top} P(s, \alpha(s)) \sigma(s)\right. \\
& +P(s, \alpha(s)) b(s)+q(s, \alpha(s))] \mathrm{d} s+\zeta(s) \mathrm{d} W(s)+\sum_{l, k=1}^{D} \xi_{l k}(s) \widetilde{N}_{l k}(s),
\end{aligned}
$$

where the last equality follows from equation (5.26).

According to (5.10), we have

$$
\begin{aligned}
0= & B(s, \alpha(s))^{\top} Y^{*}(s)+D(s, \alpha(s))^{\top} Z^{*}(s) \\
& +\left[S(s, \alpha(s))+R(s, \alpha(s)) \Theta^{*}(s, \alpha(s))\right] X^{*}(s)+R(s, \alpha(s)) v^{*}(s)+\rho(s, \alpha(s)) \\
= & B(s, \alpha(s))^{\top}\left[\eta(s)+P(s, \alpha(s)) X^{*}(s)\right] \\
& +D(s, \alpha(s))^{\top}\left\{\zeta(s)+P(s, \alpha(s))\left[C(s, \alpha(s))+D(s, \alpha(s)) \Theta^{*}(s, \alpha(s))\right] X^{*}(s)\right. \\
& \left.-P(s, \alpha(s)) D(s, \alpha(s)) v^{*}(s)-P(s, \alpha(s)) \sigma(s)\right\} \\
& +\left[S(s, \alpha(s))+R(s, \alpha(s)) \Theta^{*}(s, \alpha(s))\right] X^{*}(s)+R(s, \alpha(s)) v^{*}(s)+\rho(s, \alpha(s)) \\
= & {\left[\widehat{S}(s, \alpha(s))+\widehat{R}(s, \alpha(s)) \Theta^{*}(s, \alpha(s))\right] X^{*}(s)+\widehat{\rho}(s)+\widehat{R}(s, \alpha(s)) v^{*}(s) } \\
= & \widehat{\rho}(s)+\widehat{R}(s, \alpha(s)) v^{*}(s),
\end{aligned}
$$


where $\widehat{\rho}(s)$ is defined by (5.7). Thus, we have

$$
\widehat{\rho}(s) \in \mathcal{R}(\widehat{R}(s, \alpha(s))
$$

and

$$
v^{*}(s)=-\widehat{R}(s, \alpha(s))^{\dagger} \widehat{\rho}(s)+\left[I-\widehat{R}(s, \alpha(s))^{\dagger} \widehat{R}(s, \alpha(s))\right] \nu(s),
$$

for some $\nu(\cdot) \in L_{\mathbb{F}}^{2}\left(t, T ; \mathbb{R}^{m}\right)$. Consequently,

$$
\begin{aligned}
\widehat{S}(s, \alpha(s))^{\top} v^{*}(s) & =-\Theta^{*}(s, \alpha(s))^{\top} \widehat{R}(s, \alpha(s)) v^{*}(s) \\
& =\Theta^{*}(s, \alpha(s))^{\top} \widehat{R}(s, \alpha(s)) \widehat{R}(s, \alpha(s))^{\dagger} \widehat{\rho}(s) \\
& =-\widehat{S}(s, \alpha(s))^{\top} \widehat{R}(s, \alpha(s))^{\dagger} \widehat{\rho}(s) .
\end{aligned}
$$

Observing the definition of $\widehat{\rho}(s)$ and substituting the above equation into (5.28), we get the desired result of equation (5.5).

Sufficiency. Applying Itô's formula to $s \mapsto\langle P(s, \alpha(s)) X(s)+2 \eta(s), X(s)\rangle$ yields

$$
\begin{aligned}
J(t, x, i ; u(\cdot))=\mathbb{E}\{ & \langle P(t, i) x+2 \eta(t), x\rangle \\
& +\int_{t}^{T}[\langle P(s, \alpha(s)) \sigma(s)+2 \zeta(s), \sigma(s)\rangle+2\langle\eta(s), b(s)\rangle] \mathrm{d} s \\
& +\int_{t}^{T}[\langle\widehat{Q}(s, \alpha(s)) X(s), X(s)\rangle+\langle\widehat{R}(s, \alpha(s)) u(s)+2 \widehat{S}(s, \alpha(s)) X(s)+2 \widehat{\rho}(s), u(s)\rangle \\
& \left.\left.+2\left\langle\widehat{S}(s, \alpha(s))^{\top} \widehat{R}(s, \alpha(s))^{\dagger} \widehat{\rho}(s), X(s)\right\rangle\right] \mathrm{d} s\right\},
\end{aligned}
$$

where

$$
\begin{aligned}
\widehat{Q}(s, i):= & \dot{P}(s, i)+P(s, i) A(s, i)+A(s, i)^{\top} P(s, i) \\
& +C(s, i)^{\top} P(s, i) C(s, i)+Q(s, i)+\sum_{k=1}^{D} \lambda_{i k}(s) P(s, k) .
\end{aligned}
$$

Let $\Theta^{*}(\cdot, \alpha(\cdot))$ and $v^{*}(\cdot)$ be defined by (5.8). It is easy to verify that

$$
\begin{aligned}
\widehat{S}(s, \alpha(s)) & =-\widehat{R}(s, \alpha(s)) \Theta^{*}(s, \alpha(s)), & & \widehat{Q}(s, \alpha(s))=\Theta^{*}(s, \alpha(s))^{\top} \widehat{R}(s, \alpha(s)) \Theta^{*}(s, \alpha(s)), \\
\widehat{\rho}(s) & =-\widehat{R}(s, \alpha(s)) v^{*}(s), & & \widehat{S}(s, \alpha(s))^{\top} \widehat{R}(s, \alpha(s))^{\dagger} \widehat{\rho}(s)=\Theta^{*}(s, \alpha(s))^{\top} \widehat{R}(s, \alpha(s)) v^{*}(s) .
\end{aligned}
$$

Substituting these equations into (5.29) leads to

$$
\begin{aligned}
& J(t, x, i ; u(\cdot)) \\
& =\mathbb{E}\left\{\langle P(t, i) x+2 \eta(t), x\rangle+\int_{t}^{T}[\langle P(s, \alpha(s)) \sigma(s)+2 \zeta(s), \sigma(s)\rangle+2\langle\eta(s), b(s)\rangle] \mathrm{d} s\right. \\
& \quad+\int_{t}^{T}\left[\left\langle\Theta^{*}(s, \alpha(s))^{\top} \widehat{R}(s, \alpha(s)) \Theta^{*}(s, \alpha(s)) X(s), X(s)\right\rangle\right.
\end{aligned}
$$




$$
\begin{aligned}
& +\left\langle\widehat{R}(s, \alpha(s)) u(s)-2 \widehat{R}(s, \alpha(s))\left[\Theta^{*}(s, \alpha(s)) X(s)+v^{*}(s)\right], u(s)\right\rangle \\
& \left.\left.+2\left\langle\Theta^{*}(s, \alpha(s))^{\top} \widehat{R}(s, \alpha(s)) v^{*}(s), X(s)\right\rangle\right] \mathrm{d} s\right\} \\
=\mathbb{E}\{\langle & \langle(t, i) x+2 \eta(t), x\rangle+\int_{t}^{T}[\langle P(s, \alpha(s)) \sigma(s)+2 \zeta(s), \sigma(s)\rangle \\
& \left.+2\langle\eta(s), b(s)\rangle-\left\langle\widehat{R}(s, \alpha(s)) v^{*}(s), v^{*}(s)\right\rangle\right] \mathrm{d} s \\
& \left.+\int_{t}^{T}\left\langle\widehat{R}(s, \alpha(s))\left[u(s)-\Theta^{*}(s, \alpha(s)) X(s)-v^{*}(s)\right], u(s)-\Theta^{*}(s, \alpha(s)) X(s)-v^{*}(s)\right\rangle \mathrm{d} s\right\} \\
=J(t, & \left.x, i ; \Theta^{*}(\cdot, \alpha(\cdot)) X^{*}(\cdot)+v^{*}(\cdot)\right) \\
& +\mathbb{E} \int_{t}^{T}\left\langle\widehat{R}(s, \alpha(s))\left[u(s)-\Theta^{*}(s, \alpha(s)) X(s)-v^{*}(s)\right], u(s)-\Theta^{*}(s, \alpha(s)) X(s)-v^{*}(s)\right\rangle \mathrm{d} s .
\end{aligned}
$$

From the definition of the regular solution of Riccati equation (5.2), we know that

$$
\widehat{R}(s, \alpha(s)) \geq 0, \quad \text { a.e. } s \in[t, T] .
$$

Therefore,

$$
J\left(t, x, i ; \Theta^{*}(\cdot, \alpha(\cdot)) X^{*}(\cdot)+v^{*}(\cdot)\right) \leq J(t, x, i ; u(\cdot)), \quad \forall u(\cdot) \in \mathcal{U}[t, T],
$$

which yields that $\left(\Theta^{*}(\cdot, \alpha(\cdot)), v^{*}(\cdot)\right)$ is the closed-loop optimal strategy of Problem (M-SLQ). Finally, the representation of the value function follows from the identity

$$
\left\langle\widehat{R}(s, \alpha(s)) v^{*}(s), v^{*}(s)\right\rangle=\left\langle\widehat{R}(s, \alpha(s))^{\dagger} \widehat{\rho}(s), \widehat{\rho}(s)\right\rangle .
$$

\section{UNIFORM CONVEXITY OF THE COST FUNCTIONAL AND THE STRONGLY REGUlAR SOLUTION OF THE RICCATI EQUATION}

We first present some properties for the solution to Lyapunov equation, which play a crucial role on establishing the equivalence between uniform convexity of the cost functional and the strongly regular solution of the Riccati equation.

Lemma 6.1. Let (H1)-(H2) hold and $\Theta(\cdot, i) \in L^{2}\left(t, T ; \mathbb{R}^{m \times n}\right)$ for $i \in \mathcal{S}$. Let $P(\cdot, i) \in C\left([t, T] ; \mathbb{S}^{n}\right), i \in \mathcal{S}$ be the solution to the following Lyapunov equation:

$$
\left\{\begin{aligned}
\dot{P}(s, i) & +P(s, i) A(s, i)+A(s, i)^{\top} P(s, i)+C(s, i)^{\top} P(s, i) C(s, i) \\
& +\widehat{S}(s, i)^{\top} \Theta(s, i)+\Theta(s, i)^{\top} \widehat{S}(s, i)+\Theta(s, i)^{\top} \widehat{R}(s, i) \Theta(s, i) \\
& +Q(s, i)+\sum_{k=1}^{D} \lambda_{i k}(s) P(s, k)=0, \quad \text { a.e. } s \in[t, T], \\
P(T, i) & =G(T, i) .
\end{aligned}\right.
$$


Then for any $(t, x, i) \in[0, T) \times \mathbb{R}^{n} \times \mathcal{S}$ and $u(\cdot) \in \mathcal{U}[t, T]$, we have

$$
\begin{aligned}
& J^{0}\left(t, x, i ; \Theta(\cdot, \alpha(\cdot)) X_{0}^{\Theta, u}(\cdot ; t, x, i)+u(\cdot)\right) \\
& =\langle P(t, i) x, x\rangle+\mathbb{E} \int_{t}^{T}\left\{\left\langle T_{\alpha}^{1} u(s), u(s)\right\rangle+2\left\langle T_{\alpha}^{2} X_{0}^{\Theta, u}(s ; t, x, i), u(s)\right\rangle\right\} \mathrm{d} s .
\end{aligned}
$$

where $X_{0}^{\Theta, u}(\cdot ; t, x, i)$ is the solution of $(2.8)$ and

$$
\begin{aligned}
T_{\alpha}^{1} u(\cdot) & :=\widehat{R}(\cdot, \alpha(\cdot)) u(\cdot) \\
T_{\alpha}^{2} X_{0}^{\Theta, u}(\cdot ; t, x, i) & :=[\widehat{S}(\cdot, \alpha(\cdot))+\widehat{R}(\cdot, \alpha(\cdot)) \Theta(\cdot, \alpha(\cdot))] X_{0}^{\Theta, u}(\cdot ; t, x, i) .
\end{aligned}
$$

Proof. For any $(t, x, i) \in[0, T) \times \mathbb{R}^{n} \times \mathcal{S}$ and $u(\cdot) \in \mathcal{U}[t, T]$, let $X_{0}^{x, u}$ be the solution of (2.8) and set

$$
\begin{aligned}
T_{\alpha}^{0} X_{0}^{\Theta, u}(\cdot ; t, x, i):=[\dot{P}(\cdot, \alpha(\cdot)) & +P(\cdot, \alpha(\cdot)) A(\cdot, \alpha(\cdot))+A(\cdot, \alpha(\cdot))^{\top} P(\cdot, \alpha(\cdot))+C(\cdot, \alpha(\cdot))^{\top} P(\cdot, \alpha(\cdot)) C(\cdot, \alpha(\cdot)) \\
& +\widehat{S}(\cdot, \alpha(\cdot))^{\top} \Theta(\cdot, \alpha(\cdot))+\Theta(\cdot, \alpha(\cdot))^{\top} \widehat{S}(\cdot, \alpha(\cdot))+\Theta(\cdot, \alpha(\cdot))^{\top} \widehat{R}(\cdot, \alpha(\cdot)) \Theta(\cdot, \alpha(\cdot)) \\
& \left.+Q(\cdot, \alpha(\cdot))+\sum_{k=1}^{D} \lambda_{\alpha(\cdot) k}(\cdot) P(\cdot, k)\right] X_{0}^{\Theta, u}(\cdot ; t, x, i)
\end{aligned}
$$

Applying Itô's formula to $s \mapsto\langle P(s, \alpha(s)) X(s), X(s)\rangle$, we have

$$
\begin{aligned}
& J^{0}\left(t, x, i ; \Theta(\cdot, \alpha(\cdot)) X_{0}^{\Theta, u}(\cdot ; t, x, i)+u(\cdot)\right) \\
& =\mathbb{E}\left\{\left\langle G(T, \alpha(T)) X_{0}^{\Theta, u}(T ; t, x, i), X_{0}^{\Theta, u}(T ; t, x, i)\right\rangle+\int_{t}^{T}\left[\left\langle Q(s, \alpha(s)) X_{0}^{\Theta, u}(s ; t, x, i), X_{0}^{\Theta, u}(s ; t, x, i)\right\rangle\right.\right. \\
& \left.\left.\quad+2\left\langle S(s, \alpha(s)) X_{0}^{\Theta, u}(s ; t, x, i), u(s)\right\rangle+\langle R(s, \alpha(s)) u(s), u(s)\rangle\right] \mathrm{d} s\right\} \\
& =\langle P(t, i) x, x\rangle+\mathbb{E} \int_{t}^{T}\left\{\left\langle T_{\alpha}^{0} X_{0}^{\Theta, u}(s ; t, x, i), X_{0}^{\Theta, u}(s ; t, x, i)\right\rangle+\left\langle T_{\alpha}^{1} u(s), u(s)\right\rangle+2\left\langle T_{\alpha}^{2} X_{0}^{\Theta, u}(s ; t, x, i), u(s)\right\rangle\right\} \mathrm{d} s \\
& =\langle P(t, i) x, x\rangle+\mathbb{E} \int_{t}^{T}\left\{\left\langle T_{\alpha}^{1} u(s), u(s)\right\rangle+2\left\langle T_{\alpha}^{2} X_{0}^{\Theta, u}(s ; t, x, i), u(s)\right\rangle\right\} \mathrm{d} s .
\end{aligned}
$$

This completes the proof.

Proposition 6.2. Let (H1)-(H2) and (4.3) hold. Then for any $\Theta(\cdot, i) \in L^{2}\left(t, T ; \mathbb{R}^{m \times n}\right)$, the solution $P(\cdot, i) \in$ $C\left([t, T] ; \mathbb{S}^{n}\right)$ to the Lyapunov equation (6.1) satisfies

$$
\widehat{R}(s, i) \geqslant \lambda I, \quad \text { a.e. } \quad s \in[t, T], \quad \text { and } \quad P(s, i) \geqslant \gamma I, \quad \forall s \in[t, T],
$$

where $\gamma \in \mathbb{R}$ and appears in (4.6).

Proof. Let $\Theta(\cdot, i) \in L^{2}\left(t, T ; \mathbb{R}^{m \times n}\right)$ and let $P(\cdot, i)$ be the solution to (6.1). By (4.3) and Lemma 6.1, we have

$$
\lambda \mathbb{E} \int_{t}^{T}\left|\Theta(r, \alpha(r)) X_{0}^{\Theta, u}(r ; t, 0, i)+u(r)\right|^{2} \mathrm{~d} r \leqslant J^{0}\left(t, 0, i ; \Theta(\cdot, \alpha(\cdot)) X_{0}^{\Theta, u}(\cdot ; t, 0, i)+u(\cdot)\right)
$$




$$
=\mathbb{E} \int_{t}^{T}\left\{\langle\widehat{R}(r, \alpha(r)) u(r), u(r)\rangle+2\left\langle[\widehat{S}(r, \alpha(r))+\widehat{R}(r, \alpha(r)) \Theta(r, \alpha(r))] X_{0}^{\Theta, u}(r ; t, 0, i), u(r)\right\rangle\right\} \mathrm{d} r .
$$

Hence, for any $u(\cdot) \in \mathcal{U}[t, T]$, the following holds:

$$
\begin{aligned}
\mathbb{E} \int_{t}^{T}\left\{2\left\langle[\widehat{S}(r, \alpha(r))+(\widehat{R}(r, \alpha(r))-\lambda I) \Theta(r, \alpha(r))] X_{0}^{\Theta, u}(r ; t, 0, i), u(r)\right\rangle\right. \\
\quad+\langle(\widehat{R}(r, \alpha(r))-\lambda I) u(r), u(r)\rangle\} \mathrm{d} r \geqslant \lambda \mathbb{E} \int_{0}^{T}\left|\Theta(r, \alpha(r)) X_{0}^{\Theta, u}(r ; t, 0, i)\right|^{2} \mathrm{~d} r \geqslant 0 .
\end{aligned}
$$

Let $\Phi^{\Theta}(\cdot ; t, i):=\left(X_{0}^{\Theta, 0}\left(\cdot ; t, e_{1}, i\right), \cdots, X_{0}^{\Theta, 0}\left(\cdot ; t, e_{n}, i\right)\right)$. Then it is easy to verify that $\Phi^{\Theta}(\cdot ; t, i)$ is the solution to the following SDE for $\mathbb{R}^{n \times n}$-valued process:

$$
\left\{\begin{aligned}
& \mathrm{d} \Phi^{\Theta}(s ; t, i)= {[A(s, \alpha(s))+B(s, \alpha(s)) \Theta(s, \alpha(s))] \Phi^{\Theta}(s ; t, i) \mathrm{d} s } \\
&+[C(s, \alpha(s))+D(s, \alpha(s)) \Theta(s, \alpha(s))] \Phi^{\Theta}(s ; t, i) \mathrm{d} W(s), \quad s \in[t, T], \\
& \Phi^{\Theta}(t ; t, i)=I, \quad \alpha(t)=i .
\end{aligned}\right.
$$

Thus, $X_{0}^{\Theta, u}(\cdot ; t, 0, i)$ can be written as

$$
\begin{aligned}
X_{0}^{\Theta, u}(s ; t, 0, i) & \\
=\Phi^{\Theta}(s ; t, i) & \left\{\int_{t}^{s} \Phi^{\Theta}(v ; t, i)^{-1}[B(v, \alpha(v))-[C(v, \alpha(v))+D(v, \alpha(v)) \Theta(v, \alpha(v))] D(v, \alpha(v))] u(v) \mathrm{d} v\right. \\
& \left.+\int_{t}^{s} \Phi^{\Theta}(v ; t, i)^{-1} D(v, \alpha(v)) u(v) \mathrm{d} W(v)\right\} .
\end{aligned}
$$

Now, fix any $u_{0} \in \mathbb{R}^{m}$, take $u(r)=u_{0} \mathbf{1}_{[s, s+h]}(r)$, with $t \leqslant s \leqslant s+h \leqslant T$. Consequently, (6.3) becomes

$$
\mathbb{E} \int_{s}^{s+h}\left\{2\left\langle[\widehat{S}(r, \alpha(r))+(\widehat{R}(r, \alpha(r))-\lambda I) \Theta(r, \alpha(r))] \widehat{\Phi}(r ; t, i), u_{0}\right\rangle+\left\langle(\widehat{R}(r, \alpha(r))-\lambda I) u_{0}, u_{0}\right\rangle\right\} \mathrm{d} r \geqslant 0,
$$

where

$$
\begin{aligned}
\widehat{\Phi}(r ; t, i)=\Phi^{\Theta}(r ; t, i)\{ & \int_{s}^{r} \Phi^{\Theta}(v ; t, i)^{-1}[B(v, \alpha(v))-[C(v, \alpha(v))+D(v, \alpha(v)) \Theta(v, \alpha(v))] D(v, \alpha(v))] u_{0} \mathrm{~d} v \\
& \left.+\int_{s}^{r} \Phi^{\Theta}(v ; t, i)^{-1} D(v, \alpha(v)) u_{0} \mathrm{~d} W(v)\right\} .
\end{aligned}
$$

Dividing both sides of the above by $h$ and letting $h \rightarrow 0$, we obtain

$$
\left\langle(\widehat{R}(s, i)-\lambda I) u_{0}, u_{0}\right\rangle \geqslant 0, \quad \text { a.e. } s \in[t, T], \quad \forall u_{0} \in \mathbb{R}^{m} .
$$

Thus the first inequality in (6.2) follows. To prove the second, for any $(s, x, i) \in[t, T) \times \mathbb{R}^{n} \times \mathcal{S}$ and $u(\cdot) \in \mathcal{U}[s, T]$ and by Proposition 4.7 and Lemma 6.1, we have

$$
\begin{aligned}
\gamma|x|^{2} & \leqslant V^{0}(s, x, i) \leqslant J^{0}\left(s, x, i ; \Theta(\cdot, \alpha(\cdot)) X_{0}^{\Theta, u}(\cdot ; s, x, i)+u(\cdot)\right) \\
& =\langle P(s, i) x, x\rangle+\mathbb{E} \int_{s}^{T}\{\langle\widehat{R}(r, \alpha(r)) u(r), u(r)\rangle
\end{aligned}
$$




$$
\left.+2\left\langle[\widehat{S}(r, \alpha(r))+\widehat{R}(r, \alpha(r)) \Theta(r, \alpha(r))] X_{0}^{\Theta, u}(r ; t, 0, i), u(r)\right\rangle\right\} \mathrm{d} r .
$$

In particular, by taking $u(\cdot)=0$ in the above, we obtain

$$
\langle P(s, i) x, x\rangle \geqslant \gamma|x|^{2}, \quad \forall(s, x, i) \in[t, T] \times \mathbb{R}^{n} \times \mathcal{S},
$$

and the second inequality therefore follows.

Now we further prove the equivalence between the uniform convexity of the cost functional and the strongly regular solution of Riccati equation.

Theorem 6.3. Let (H1)-(H2) hold. Then the following statements are equivalent:

(i) The map $u(\cdot) \mapsto J^{0}(t, 0, i ; u(\cdot))$ is uniformly convex, i.e., there exists a $\lambda>0$ such that (4.3) holds.

(ii) The Riccati equation (5.2) admit a strongly regular solution $P(\cdot, \cdot) \in C\left([t, T] \times \mathcal{S} ; \mathbb{S}^{n}\right)$.

Proof. (i) $\Rightarrow$ (ii). Let $P_{0}(\cdot, \cdot)$ be the solution of

$$
\left\{\begin{aligned}
\dot{P}_{0}(s, i) & +P_{0}(s, i) A(s, i)+A(s, i)^{\top} P_{0}(s, i) \\
& +C(s, i)^{\top} P_{0}(s, i) C(s, i)+Q(s, i)+\sum_{k=1}^{D} \lambda_{i k}(s) P_{0}(s, k)=0, \quad \text { a.e. } s \in[t, T], \\
P_{0}(T, i) & =G(T, i) .
\end{aligned}\right.
$$

Applying Proposition 6.2 with $\Theta(\cdot)=0$, we obtain that

$$
\widehat{R}(s, i) \geqslant \lambda I, \quad P_{0}(s, i) \geqslant \gamma I, \quad \text { a.e. } s \in[t, T] .
$$

Next, inductively, for $n=0,1,2, \ldots$, we set

$$
\left\{\begin{array}{l}
\Theta_{n}(s, i)=-\widehat{R}(s, i)^{-1}\left[B(s, i)^{\top} P_{n}(s, i)+D(s, i)^{\top} P_{n}(s, i) C(s, i)+S(s, i)\right] \\
A_{n}(s, i)=A(s, i)+B(s, i) \Theta_{n}(s, i) \\
C_{n}(s, i)=C(s, i)+D(s, i) \Theta_{n}(s, i)
\end{array}\right.
$$

and let $P_{n+1}$ be the solution of the iteration

$$
\left\{\begin{array}{l}
\dot{P}_{n+1}(s, i)+P_{n+1}(s, i) A_{n}(s, i)+A_{n}(s, i)^{\top} P_{n+1}(s, i) \\
\quad+C_{n}(s, i)^{\top} P_{n+1}(s, i) C_{n}(s, i)+Q_{n}(s, i)+\sum_{k=1}^{D} \lambda_{i k}(s) P_{n+1}(s, k)=0, \quad \text { a.e. } s \in[t, T], \\
P_{n+1}(T, i)=G(T, i) .
\end{array}\right.
$$

By Proposition 6.2, we have

$$
\left\{\begin{array}{l}
R(s, i)+D(s, i)^{\top} P_{n+1}(s, i) D(s, i) \geqslant \lambda I \\
P_{n+1}(s, i) \geqslant \gamma I, \quad \text { a.e. } s \in[t, T], \quad n=0,1,2, \cdots .
\end{array}\right.
$$

We further claim that $\left\{P_{n}(s, i)\right\}_{n=1}^{\infty}$ converges uniformly in $C\left([t, T] ; \mathbb{S}^{n}\right)$. To show this, let

$$
\Delta_{n}(s, i) \triangleq P_{n}(s, i)-P_{n+1}(s, i), \quad \Lambda_{n}(s, i) \triangleq \Theta_{n-1}(s, i)-\Theta_{n}(s, i), \quad n \geqslant 1 .
$$


Then for $n \geqslant 1$, we have

$$
\begin{aligned}
-\dot{\Delta}_{n}(s, i)= & \dot{P}_{n+1}(s, i)-\dot{P}_{n}(s, i) \\
= & P_{n}(s, i) A_{n-1}(s, i)+A_{n-1}(s, i)^{\top} P_{n}(s, i)+C_{n-1}(s, i)^{\top} P_{n}(s, i) C_{n-1}(s, i) \\
& +\Theta_{n-1}(s, i)^{\top} R(s, i) \Theta_{n-1}(s, i)+S(s, i)^{\top} \Theta_{n-1}(s, i)+\Theta_{n-1}(s, i)^{\top} S(s, i) \\
& -P_{n+1}(s, i) A_{n}(s, i)-A_{n}(s, i)^{\top} P_{n+1}(s, i)-C_{n}(s, i)^{\top} P_{n+1}(s, i) C_{n}(s, i) \\
& -\Theta_{n}(s, i)^{\top} R(s, i) \Theta_{n}(s, i)-S(s, i)^{\top} \Theta_{n}(s, i)-\Theta_{n}(s, i)^{\top} S(s, i)+\sum_{k=1}^{D} \lambda_{i k}(s) \Delta_{n}(s, k) \\
= & \Delta_{n}(s, i) A_{n}(s, i)+A_{n}(s, i)^{\top} \Delta_{n}(s, i)+C_{n}(s, i)^{\top} \Delta_{n}(s, i) C_{n}(s, i) \\
& +P_{n}(s, i)\left(A_{n-1}(s, i)-A_{n}(s, i)\right)+\left(A_{n-1}(s, i)-A_{n}(s, i)\right)^{\top} P_{n}(s, i) \\
& +C_{n-1}(s, i)^{\top} P_{n}(s, i) C_{n-1}(s, i)-C_{n}(s, i)^{\top} P_{n}(s, i) C_{n}(s, i) \\
& +\Theta_{n-1}(s, i)^{\top} R(s, i) \Theta_{n-1}(s, i)-\Theta_{n}(s, i)^{\top} R(s, i) \Theta_{n}(s, i) \\
& +S(s, i)^{\top} \Lambda_{n}(s, i)+\Lambda_{n}(s, i)^{\top} S(s, i)+\sum_{k=1}^{D} \lambda_{i k}(s) \Delta_{n}(s, k) .
\end{aligned}
$$

By (6.5), we have the following:

$$
\left\{\begin{array}{l}
A_{n-1}(s, i)-A_{n}(s, i)=B(s, i) \Lambda_{n}(s, i), \\
C_{n-1}(s, i)-C_{n}(s, i)=D(s, i) \Lambda_{n}(s, i), \\
C_{n-1}(s, i)^{\top} P_{n}(s, i) C_{n-1}(s, i)-C_{n}(s, i)^{\top} P_{n}(s, i) C_{n}(s, i) \\
=\Lambda_{n}(s, i)^{\top} D(s, i)^{\top} P_{n}(s, i) D(s, i) \Lambda_{n}(s, i)+C_{n}(s, i)^{\top} P_{n}(s, i) D(s, i) \Lambda_{n}(s, i) \\
\quad+\Lambda_{n}(s, i)^{\top} D(s, i)^{\top} P_{n}(s, i) C_{n}(s, i), \\
\Theta_{n-1}(s, i)^{\top} R(s, i) \Theta_{n-1}(s, i)-\Theta_{n}(s, i)^{\top} R(s, i) \Theta_{n}(s, i) \\
=\Lambda_{n}(s, i)^{\top} R(s, i) \Lambda_{n}(s, i)+\Lambda_{n}(s, i)^{\top} R(s, i) \Theta_{n}(s, i)+\Theta_{n}(s, i)^{\top} R(s, i) \Lambda_{n}(s, i) .
\end{array}\right.
$$

Note that

$$
\begin{aligned}
& B(s, i)^{\top} P_{n}(s, i)+D(s, i)^{\top} P_{n}(s, i) C_{n}(s, i)+R(s, i) \Theta_{n}(s, i)+S(s, i) \\
& =B(s, i)^{\top} P_{n}(s, i)+D(s, i)^{\top} P_{n}(s, i) C(s, i)+S(s, i)+\left(R(s, i)+D(s, i)^{\top} P_{n}(s, i) D(s, i)\right) \Theta_{n}(s, i)=0 .
\end{aligned}
$$

Thus, substituting (6.8) into (6.7) leads to

$$
\begin{aligned}
- & {\left[\dot{\Delta}_{n}(s, i)+\Delta_{n}(s, i) A_{n}(s, i)+A_{n}(s, i)^{\top} \Delta_{n}(s, i)+C_{n}(s, i)^{\top} \Delta_{n}(s, i) C_{n}(s, i)+\sum_{k=1}^{D} \lambda_{i k}(s) \Delta_{n}(s, k)\right] } \\
= & P_{n}(s, i) B(s, i) \Lambda_{n}(s, i)+\Lambda_{n}(s, i)^{\top} B(s, i)^{\top} P_{n}(s, i)+\Lambda_{n}(s, i)^{\top} D(s, i)^{\top} P_{n}(s, i) D(s, i) \Lambda_{n}(s, i) \\
& +C_{n}(s, i)^{\top} P_{n}(s, i) D(s, i) \Lambda_{n}(s, i)+\Lambda_{n}(s, i)^{\top} D(s, i)^{\top} P_{n}(s, i) C_{n}(s, i)+\Lambda_{n}(s, i)^{\top} R(s, i) \Lambda_{n}(s, i) \\
& +\Lambda_{n}(s, i)^{\top} R(s, i) \Theta_{n}(s, i)+\Theta_{n}(s, i)^{\top} R(s, i) \Lambda_{n}(s, i)+S(s, i)^{\top} \Lambda_{n}(s, i)+\Lambda_{n}(s, i)^{\top} S(s, i) \\
= & \Lambda_{n}(s, i)^{\top}\left[R(s, i)+D(s, i)^{\top} P_{n}(s, i) D(s, i)\right] \Lambda_{n}(s, i) \\
& +\left[P_{n}(s, i) B(s, i)+C_{n}(s, i)^{\top} P_{n}(s, i) D(s, i)+\Theta_{n}(s, i)^{\top} R(s, i)+S(s, i)^{\top}\right] \Lambda_{n}(s, i) \\
& +\Lambda_{n}(s, i)^{\top}\left[B(s, i)^{\top} P_{n}(s, i)+D(s, i)^{\top} P_{n}(s, i) C_{n}(s, i)+R(s, i) \Theta_{n}(s, i)+S(s, i)\right]
\end{aligned}
$$




$$
=\Lambda_{n}(s, i)^{\top}\left[R(s, i)+D(s, i)^{\top} P_{n}(s, i) D(s, i)\right] \Lambda_{n}(s, i) \geqslant 0 .
$$

Noting that $\Delta_{n}(T, i)=0$, and using Proposition 3.2 and (6.6), we obtain

$$
P_{1}(s, i) \geqslant P_{n}(s, i) \geqslant P_{n+1}(s, i) \geqslant \alpha I, \quad \forall s \in[t, T], \quad \forall n \geqslant 1
$$

Therefore, the sequence $\left\{P_{n}(s, i)\right\}_{n=1}^{\infty}$ is uniformly bounded. Consequently, there exists a constant $K>0$ such that (noting (6.6))

$$
\left\{\begin{array}{l}
\left|P_{n}(s, i)\right|,\left|R_{n}(s, i)\right| \leqslant K \\
\left|\Theta_{n}(s, i)\right| \leqslant K(|B(s, i)|+|C(s, i)|+|S(s, i)|), \\
\left|A_{n}(s, i)\right| \leqslant|A(s, i)|+K|B(s, i)|(|B(s, i)|+|C(s, i)|+|S(s, i)|), \\
\left|C_{n}(s, i)\right| \leqslant|C(s, i)|+K(|B(s, i)|+|C(s, i)|+|S(s, i)|),
\end{array} \quad \text { a.e. } s \in[t, T], \forall i \in \mathcal{S}, \forall n \geqslant 0\right.
$$

where $R_{n}(s, i) \triangleq R(s, i)+D^{\top}(s, i) P_{n}(s, i) D(s, i)$. Since

$$
\begin{aligned}
\Lambda_{n}(s, i)= & \Theta_{n-1}(s, i)-\Theta_{n}(s, i) \\
= & R_{n}(s, i)^{-1} D(s, i)^{\top} \Delta_{n-1}(s, i) D(s, i) R_{n-1}(s, i)^{-1} \widehat{S}_{n}(s, i) \\
& -R_{n-1}(s, i)^{-1}\left[B(s, i)^{\top} \Delta_{n-1}(s, i)+D(s, i)^{\top} \Delta_{n-1}(s, i) C(s, i)\right],
\end{aligned}
$$

where $\widehat{S}_{n}(s, i):=B(s, i)^{\top} P_{n}(s, i)+D(s, i)^{\top} P_{n}(s, i) C(s, i)+S(s, i)$, one has

$$
\begin{aligned}
\left|\Lambda_{n}(s, i)^{\top} R_{n}(s, i) \Lambda_{n}(s, i)\right| & \leqslant\left(\left|\Theta_{n}(s, i)\right|+\left|\Theta_{n-1}(s, i)\right|\right)\left|R_{n}(s, i)\right|\left|\Theta_{n-1}(s, i)-\Theta_{n}(s, i)\right| \\
& \leqslant K(|B(s, i)|+|C(s, i)|+|S(s, i)|)^{2}\left|\Delta_{n-1}(s, i)\right| .
\end{aligned}
$$

Equation (6.9), together with $\Delta_{n}(T, i)=0$, implies that

$$
\begin{gathered}
\Delta_{n}(s, i)=\int_{s}^{T}\left[\Delta_{n}(r, i) A_{n}(r, i)+A_{n}(r, i)^{\top} \Delta_{n}(r, i)+C_{n}(r, i)^{\top} \Delta_{n}(r, i) C_{n}(r, i)\right. \\
\left.+\Lambda_{n}(r, i)^{\top} R_{n}(r, i) \Lambda_{n}(r, i)+\sum_{k=1}^{D} \lambda_{i k}(r) \Delta_{n}(r, k)\right] \mathrm{d} r .
\end{gathered}
$$

Making use of (6.12), together with (6.10), we get

$$
\left|\Delta_{n}(s, i)\right| \leqslant \int_{s}^{T} \varphi(r)\left[\left|\sum_{k=1}^{D} \Delta_{n}(r, k)\right|+\left|\Delta_{n-1}(r, i)\right|\right] \mathrm{d} r, \quad \forall s \in[t, T], \quad \forall n \geqslant 1,
$$

where $\varphi(\cdot)$ is a nonnegative integrable function independent of $\Delta_{n}(\cdot, \cdot)$. Let

$$
\left\|\Delta_{n}(s)\right\|:=\max _{k=1}^{D}\left|\Delta_{n}(s, k)\right|
$$


Hence, from (6.13), we have

$$
\left\|\Delta_{n}(s)\right\| \leqslant \int_{s}^{T} \varphi(r)\left[\left\|\Delta_{n}(r)\right\|+\left\|\Delta_{n-1}(r)\right\|\right] \mathrm{d} r, \quad \forall s \in[t, T], \quad \forall n \geqslant 1,
$$

By Gronwall's inequality, we obtain

$$
\left\|\Delta_{n}(s)\right\| \leqslant e^{\int_{0}^{T} \varphi(r) \mathrm{d} r} \int_{s}^{T} \varphi(r)\left\|\Delta_{n-1}(r)\right\| \mathrm{d} r \equiv c \int_{s}^{T} \varphi(r)\left\|\Delta_{n-1}(r)\right\| \mathrm{d} r .
$$

Set

$$
a \triangleq \max _{t \leqslant s \leqslant T}\left\|\Delta_{0}(s)\right\|
$$

By induction, we deduce that

$$
\left\|\Delta_{n}(s)\right\| \leqslant a \frac{c^{n}}{n !}\left(\int_{s}^{T} \varphi(r) \mathrm{d} r\right)^{n}, \quad \forall s \in[t, T]
$$

which implies the uniform convergence of $\left\{P_{n}(\cdot, \cdot)\right\}_{n=1}^{\infty}$. We denote $P(\cdot, \cdot)$ the limit of $\left\{P_{n}(\cdot, \cdot)\right\}_{n=1}^{\infty}$, then (noting (6.6))

$$
R(s, i)+D(s, i)^{\top} P(s, i) D(s, i)=\lim _{n \rightarrow \infty} R(s, i)+D(s, i)^{\top} P_{n}(s, i) D(s, i) \geqslant \epsilon I, \quad \text { a.e. } \quad s \in[t, T],
$$

and as $n \rightarrow \infty$,

$$
\begin{cases}\Theta_{n}(s, i) \rightarrow-\widehat{R}(s, i) \widehat{S}(s, i) \equiv \Theta(s, i) & \text { in } L^{2} \\ A_{n}(s, i) \rightarrow A(s, i)+B(s, i) \Theta(s, i) & \text { in } L^{1} \\ C_{n}(s, i) \rightarrow C(s, i)+D(s, i) \Theta(s, i) & \text { in } L^{2}\end{cases}
$$

Therefore, $P(\cdot, \cdot)$ satisfies the following equation:

$$
\left\{\begin{array}{l}
\dot{P}(s, i)+P(s, i)[A(s, i)+B(s, i) \Theta(s, i)]+[A(s, i)+B(s, i) \Theta(s, i)]^{\top} P(s, i) \\
\quad+[C(s, i)+D(s, i) \Theta(s, i)]^{\top} P(s, i)[C(s, i)+D(s, i) \Theta(s, i)]+\Theta(s, i)^{\top} R(s, i) \Theta(s, i) \\
\quad+S(s, i)^{\top} \Theta(s, i)+\Theta(s, i)^{\top} S(s, i)+Q(s, i)+\sum_{k=1}^{D} \lambda_{i k}(s) P(s, k)=0, \quad \text { a.e. } s \in[t, T], \\
P(T, i)=G(T, i),
\end{array}\right.
$$

which is equivalent to $(5.2)$.

(ii) $\Rightarrow$ (i). Let $P(\cdot, \cdot)$ be the strongly regular solution of $(5.2)$. Then there exists $\epsilon>0$ such that

$$
\widehat{R}(s, i) \geqslant \epsilon I, \quad \text { a.e. } s \in[t, T] .
$$

Set

$$
\Theta(s, \alpha(s)) \triangleq-\widehat{R}(s, \alpha(s)) \widehat{S}(s, \alpha(s)) \in L^{2}\left(0, T ; \mathbb{R}^{m \times n}\right) .
$$


For any $u(\cdot) \in \mathcal{U}[t, T]$, let $X_{0}^{u}(\cdot ; t, 0, i)$ be the solution of

$$
\left\{\begin{aligned}
& \mathrm{d} X_{0}^{u}(s ; t, 0, i)=\left[A(s, \alpha(s)) X_{0}^{u}(s ; t, 0, i)+B(s, \alpha(s)) u(s)\right] \mathrm{d} s \\
&+\left[C(s, \alpha(s)) X_{0}^{u}(s ; t, 0, i)+D(s, \alpha(s)) u(s)\right] \mathrm{d} W(s), \quad s \in[t, T] \\
& X_{0}^{0, u}(t)=0, \quad \alpha(t)=i
\end{aligned}\right.
$$

Applying Itô's formula to $s \mapsto\left\langle P(s, \alpha(s)) X_{0}^{u}(s ; t, 0, i), X_{0}^{u}(s ; t, 0, i)\right\rangle$, we have

$$
\begin{aligned}
& J^{0}(t, 0 ; u(\cdot)) \\
& =\mathbb{E}\left\{\left\langle G(T, \alpha(T)) X_{0}^{u}(T ; t, 0, i), X_{0}^{u}(T ; t, 0, i)\right\rangle+\int_{t}^{T}\left[\left\langle Q(s, \alpha(s)) X_{0}^{u}(s ; t, 0, i), X_{0}^{u}(s ; t, 0, i)\right\rangle\right.\right. \\
& \left.\left.+2\left\langle S(s, \alpha(s)) X_{0}^{u}(s ; t, 0, i), u(s)\right\rangle+\langle R(s, \alpha(s)) u(s), u(s)\rangle\right] \mathrm{d} s\right\} \\
& =\mathbb{E} \int_{t}^{T}\left[\left\langle\dot{P}(s, \alpha(s)) X_{0}^{u}(s ; t, 0, i), X_{0}^{u}(s ; t, 0, i)\right\rangle\right. \\
& +\left\langle P(s, \alpha(s))\left[A(s, \alpha(s)) X_{0}^{u}(s ; t, 0, i)+B(s, \alpha(s)) u(s)\right], X_{0}^{u}(s ; t, 0, i)\right\rangle \\
& +\left\langle P(s, \alpha(s)) X_{0}^{u}(s ; t, 0, i), A(s, \alpha(s)) X_{0}^{u}(s ; t, 0, i)+B(s, \alpha(s)) u(s)\right\rangle \\
& +\left\langle P(s, \alpha(s))\left[C(s, \alpha(s)) X_{0}^{u}(s ; t, 0, i)+D(s, \alpha(s)) u(s)\right], C(s, \alpha(s)) X_{0}^{u}(s ; t, 0, i)+D(s, \alpha(s)) u(s)\right\rangle \\
& +\left\langle Q(s, \alpha(s)) X_{0}^{u}(s ; t, 0, i), X_{0}^{u}(s ; t, 0, i)\right\rangle+2\left\langle S(s, \alpha(s)) X_{0}^{u}(s ; t, 0, i), u(s)\right\rangle \\
& \left.+\langle R(s, \alpha(s)) u(s), u(s)\rangle+\left\langle\sum_{k=1}^{D} \lambda_{\alpha(s-), k}(s) P(s, k) X_{0}^{u}(s ; t, 0, i), X_{0}^{u}(s ; t, 0, i)\right\rangle\right] \mathrm{d} s \\
& =\mathbb{E} \int_{t}^{T}\left[\left\langle\widehat{Q}(s, \alpha(s)) X_{0}^{u}(s ; t, 0, i), X_{0}^{u}(s ; t, 0, i)\right\rangle+2\left\langle\widehat{S}(s, \alpha(s)) X_{0}^{u}(s ; t, 0, i), u(s)\right\rangle+\langle\widehat{R}(s, \alpha(s)) u(s), u(s)\rangle\right] \mathrm{d} s \\
& =\mathbb{E} \int_{t}^{T}\left[\left\langle\Theta(s, \alpha(s))^{\top} \widehat{R}(s, \alpha(s)) \Theta(s, \alpha(s)) X_{0}^{u}(s ; t, 0, i), X_{0}^{u}(s ; t, 0, i)\right\rangle\right. \\
& \left.-2\left\langle\widehat{R}(s, \alpha(s)) \Theta(s, \alpha(s)) X_{0}^{u}(s ; t, 0, i), u(s)\right\rangle+\langle\widehat{R}(s, \alpha(s)) u(s), u(s)\rangle\right] \mathrm{d} s \\
& =\mathbb{E} \int_{t}^{T}\left\langle\left[\widehat{R}(s, \alpha(s))\left[u(s)-\Theta(s, \alpha(s)) X_{0}^{u}(s ; t, 0, i)\right], u(s)-\Theta(s, \alpha(s)) X_{0}^{u}(s ; t, 0, i)\right\rangle \mathrm{d} s .\right.
\end{aligned}
$$

Noting (6.15) and making use of Lemma 4.5, we obtain

$$
\begin{aligned}
J^{0}(t, 0 ; u(\cdot)) & =\mathbb{E} \int_{t}^{T}\left\langle\widehat{R}(s, \alpha(s))\left[u(s)-\Theta(s, \alpha(s)) X_{0}^{u}(s ; t, 0, i)\right], u(s)-\Theta(s, \alpha(s)) X_{0}^{u}(s ; t, 0, i)\right\rangle \mathrm{d} s \\
& \geqslant \lambda \gamma \mathbb{E} \int_{t}^{T}|u(s)|^{2} \mathrm{~d} s, \quad \forall u(\cdot) \in \mathcal{U}[t, T],
\end{aligned}
$$

for some $\gamma>0$. Then (i) holds.

Remark 6.4. From the first part of the proof of Theorem 4.6, we see that if (4.3) holds, then the strongly regular solution of (5.2) satisfies (5.4) with the same constant $\lambda>0$.

Combining Theorem 5.2 and Theorem 6.3, we obtain the following corollary. 
Corollary 6.5. Let $P(\cdot, \cdot)$ be the unique strongly regular solution of $(5.2)$ with $(\eta(\cdot), \zeta(\cdot), \xi(\cdot))$ being the adapted solution of (5.5). Suppose that (H1)-(H2) and (4.3) hold. Then Problem (M-SLQ) is uniquely open-loop solvable at any $(t, x, i) \in[0, T) \times \mathbb{R}^{n} \times \mathcal{S}$ with the open-loop optimal control $u^{*}(\cdot)$ being of a state feedback form:

$$
u^{*}(\cdot)=-\widehat{R}(\cdot, \alpha(\cdot))^{-1} \widehat{S}(\cdot, \alpha(\cdot)) X^{*}(\cdot)-\widehat{R}(\cdot, \alpha(\cdot))^{-1} \widehat{\rho}(\cdot)
$$

where $\widehat{R}(\cdot, \cdot)$ and $\widehat{\rho}(\cdot)$ are defined by (5.1) and (5.7) respectively, and $X^{*}(\cdot)$ is the solution of the following closed-loop system:

$$
\left\{\begin{aligned}
& \mathrm{d} X^{*}(s)=\left\{\left[A(s, \alpha(s))-B(s, \alpha(s)) \widehat{R}(s, \alpha(s))^{-1} \widehat{S}(s, \alpha(s))\right] X^{*}(s)\right. \\
&\left.-B(s, \alpha(s)) \widehat{R}(s, \alpha(s))^{-1} \widehat{\rho}(s)+b(s)\right\} \mathrm{d} s \\
&+\left\{\left[C(s, \alpha(s))-D(s, \alpha(s)) \widehat{R}(s, \alpha(s))^{-1} \widehat{S}(s, \alpha(s))\right] X^{*}(s)\right. \\
&\left.-D(s, \alpha(s)) \widehat{R}(s, \alpha(s))^{-1} \widehat{\rho}(s)+\sigma(s)\right\} \mathrm{d} W(s), \quad s \in[t, T], \\
& X^{*}(t)=x, \quad \alpha(t)=i .
\end{aligned}\right.
$$

Proof. By Theorem 6.3, the Riccati equation (5.2) admit a unique strongly regular solution $P(\cdot, \cdot) \in$ $C\left([t, T] \times \mathcal{S} ; \mathbb{S}^{n}\right)$. Hence, the adapted solution $(\eta(\cdot), \zeta(\cdot), \xi(\cdot))$ of $(5.5)$ satisfies (5.6) automatically. Now applying Theorem 5.2 and noting the remark right after Definition 2.3, we get the desired result.

Remark 6.6. Under the assumptions of Corollary 6.5, when $b(\cdot), \sigma(\cdot), g(\cdot, \cdot), q(\cdot, \cdot), \rho(\cdot, \cdot)=0$, the adapted solution of $(5.5)$ is $(\eta(\cdot), \zeta(\cdot), \xi(\cdot)) \equiv(0,0,0)$. Thus, for Problem (M - SLQ $)^{0}$, the unique optimal control $u^{*}(\cdot)$ at initial pair $(t, x, i) \in[0, T) \times \mathbb{R}^{n} \times \mathcal{S}$ is given by

$$
u^{*}(\cdot)=-\widehat{R}(\cdot, \alpha(\cdot))^{-1} \widehat{S}(\cdot, \alpha(\cdot)) X^{*}(\cdot),
$$

with $P(\cdot, \cdot)$ being the unique strongly regular solution of $(5.2)$ and $X^{*}(\cdot)$ being the solution of the following closed-loop system:

$$
\left\{\begin{array}{l}
\mathrm{d} X^{*}(s)=\left[A(s, \alpha(s))-B(s, \alpha(s)) \widehat{R}(s, \alpha(s))^{-1} \widehat{S}(s, \alpha(s))\right] X^{*}(s) \mathrm{d} s \\
\quad+\left[C(s, \alpha(s))-D(s, \alpha(s)) \widehat{R}(s, \alpha(s))^{-1} \widehat{S}(s, \alpha(s))\right] X^{*}(s) \mathrm{d} W(s), \quad s \in[t, T], \\
X^{*}(t)=x, \quad \alpha(t)=i .
\end{array}\right.
$$

Moreover, by (5.9), the value function of Problem (M - SLQ $)^{0}$ is given by

$$
V^{0}(t, x, i)=\langle P(t, i) x, x\rangle, \quad(t, x, i) \in[0, T] \times \mathbb{R}^{n} \times \mathcal{S} .
$$

\section{ExAMPLE}

In this section, we present an example of M-SLQ problem which is open-loop solvable but not closed-loop solvable.

Example 7.1. Consider the following one-dimensional controlled Markovian regime switching state process

$$
\left\{\begin{aligned}
\mathrm{d} X^{u}(s ; t, x, i) & =\left[-2 e^{\alpha(s)} X^{u}(s ; t, x, i)+e^{\alpha(s)} u(s)\right] \mathrm{d} s+\left[2 e^{\alpha(s) / 2} X^{u}(s ; t, x, i)\right] \mathrm{d} W(s), \\
X^{u}(t ; t, x, i) & =x, \quad \alpha(t)=i,
\end{aligned}\right.
$$


and the cost functional is defined by

$$
J(t, x, i ; u(\cdot)) \triangleq \mathbb{E}\left[|X(T ; t, x, i)|^{2}\right] .
$$

Noting that $Q(\cdot, i)=0, R(\cdot, i)=0, D(\cdot, i)=0$ for every $i \in \mathcal{S}$ and $0^{\dagger}=0$, it is easy to see that Riccati equation (5.2) becomes

$$
\left\{\begin{array}{l}
\dot{P}(s, i)+\sum_{k=1}^{D} \lambda_{i k}(s) P(s, k)=0, \quad \text { a.e. } s \in[t, T], \\
P(T, i)=1 .
\end{array}\right.
$$

Clearly, the unique solution of the above ordinary differential equation system is

$$
P(s, i) \equiv 1, \text { for }(s, i) \in[t, T] \times \mathcal{S} .
$$

Thus, for any $(s, i) \in[t, T] \times \mathcal{S}$, we have

$$
\begin{aligned}
\mathcal{R}(\widehat{S}(s, i))=\mathcal{R}\left(e^{i}\right) & =\mathcal{R}(1)=\mathbb{R}, \quad \Longrightarrow \quad \mathcal{R}(\widehat{S}(s, i)) \nsubseteq \mathcal{R}(\widehat{R}(s, i)) . \\
\mathcal{R}(\widehat{R}(s, i)) & =\mathcal{R}(0)=\{0\}, \quad
\end{aligned}
$$

Now from Theorem 5.2, we can deduce that this M-SLQ problem is not closed-loop solvable.

In what follows, we show that this M-SLQ problem is open-loop solvable. In fact, if we let $u^{*}(\cdot)$ be the control defined by

$$
u^{*}(s) \triangleq \frac{x}{t-T} \exp \left\{-\alpha(s)-4 \int_{t}^{s} e^{\alpha(r)} \mathrm{d} r+2 \int_{t}^{s} e^{\alpha(r) / 2} \mathrm{~d} W(r)\right\}
$$

and $X^{*}(s ; t, x, i)$ be the controlled state process corresponding to the initial state $(t, x, i)$ and the control $u^{*}(\cdot)$, then by the variation of constant formula, we have

$$
\begin{aligned}
X^{*}(s ; t, x, i)= & \exp \left\{-4 \int_{t}^{s} e^{\alpha(r)} \mathrm{d} r+2 \int_{t}^{s} e^{\alpha(r) / 2} \mathrm{~d} W(r)\right\} \\
& \cdot\left[x+\int_{t}^{s} \exp \left\{4 \int_{t}^{r} e^{\alpha(v)} \mathrm{d} v-2 \int_{t}^{r} e^{\alpha(v) / 2} \mathrm{~d} W(v)\right\} e^{\alpha(r)} u^{*}(r) \mathrm{d} r\right] \\
= & x \exp \left\{-4 \int_{t}^{s} e^{\alpha(r)} \mathrm{d} r+2 \int_{t}^{s} e^{\alpha(r) / 2} \mathrm{~d} W(r)\right\}\left[1+\frac{s-t}{t-T}\right], s \in[t, T] .
\end{aligned}
$$

Hence,

$$
J\left(t, x, i ; u^{*}(\cdot)\right)=\mathbb{E}\left[\left|X^{*}(T)\right|^{2}\right]=0 \leq \mathbb{E}\left[|X(T ; t, x, i)|^{2}\right]=J(t, x, i ; u(\cdot)),
$$

which implies the open-loop solvability of the M-SLQ problem and $u^{*}(\cdot)$ is open-loop optimal for the initial state $(t, x, i)$.

Acknowledgements. The authors would like to thank referees and editors for insightful comments that have significantly improved the paper. 


\section{REFERENCES}

[1] J.-M. Bismut, Linear quadratic optimal stochastic control with random coefficients. SIAM J. Control Optim. 14 (1976) 419-444.

[2] S. Chen, X. Li and X. Zhou, Stochastic linear quadratic regulators with indefinite control weight costs. SIAM J. Control Optim. 36 (1998) 1685-1702.

[3] S. Chen and J. Yong, Stochastic linear quadratic optimal control problems. Appl. Math. Optim. 43 (2001) $21-45$.

[4] Y. Hu and B. Oksendal, Partial information linear quadratic control for jump diffusions. SIAM J. Control Optim. 47 (2008) $1744-1761$.

[5] J. Huang and Z. Yu, Solvability of indefinite stochastic Riccati equations and linear quadratic optimal control problems. Syst. Control Lett. 68 (2014) 68-75.

[6] Y. Ji and H. Chizeck, Jump linear quadratic Gaussian control in continuous time. IEEE Trans. Autom. Control. 37 (1992) $1884-1892$.

[7] Y. Ji and H. Chizeck, Controllability, stabilizability, and continuous-time Markovian jump linear quadratic control. IEEE Trans. Autom. Control. 35 (1990) 777-788.

[8] M. Kohlmann and S. Tang, New developments in backward stochastic riccati equations and their applications, in Mathematical Finance, edited by M. Kohlmann, S. Tang. Birkhäuser, Basel (2001) 194-214.

[9] M. Kohlmann and S. Tang, Global adapted solution of one-dimensional backward stochastic riccati equations, with application to the mean-variance hedging. Stoch. Process. Appl. 97 (2002) 255-288.

[10] M. Kohlmann and S. Tang, Minimization of risk and linear quadratic optimal control theory. SIAM J. Control Optim. 42 (2003) 1118-1142.

[11] M. Kohlmann and S. Tang, Multidimensional backward stochastic Riccati equations and applications. SIAM J. Control Optim. 41 (2003) 1696-1721.

[12] H. Kushner, Optimal stochastic control. IRE Trans. Autom. Control. 7 (1962) 120-122.

[13] N. Li, Z. Wu and Z. Yu, Indefinite stochastic linear-quadratic optimal control problems with random jumps and related stochastic Riccati equations. Sci. China Math. 61 (2018) 563-576.

[14] X. Li and X. Zhou, Indefinite stochastic LQ controls with Markovian jumps in a finite time horizon. Commun. Inf. Syst. 2 (2002) 265-282.

[15] X. Li, X. Zhou and M. Rami, Indefinite stochastic LQ control with jumps, in Proceedings of the 40th IEEE Conference on Decision and Control (Cat. No.01CH37228). IEEE (2001).

[16] X. Li, X. Zhou and M.A. Rami, Indefinite stochastic linear quadratic control with Markovian jumps in infinite time horizon. J. Global Optim. 27 (2003) 149-175.

[17] Y. Liu, G. Yin and X.Y. Zhou, Near-optimal controls of random-switching LQ problems with indefinite control weight costs. Automatica. 41 (2005) 1063-1070.

[18] Q. Lv, T. Wang and X. Zhang, Characterization of optimal feedback for stochastic linear quadratic control problems. Probab. Uncert. Quant. Risk. 2 (2017) Article number: 11.

[19] H. Mei and J. Yong, Equilibrium strategies for time-inconsistent stochastic switching systems. ESAIM: COCV 25 (2019) Article number: 64 .

[20] J. Sun, X. Li and J. Yong, Open-loop and closed-loop solvabilities for stochastic linear quadratic optimal control problems. SIAM J. Control Optim. 54 (2016) 2274-2308.

[21] J. Sun, J. Xiong and J. Yong, Stochastic linear-quadratic optimal control problems with random coefficients: closed-loop representation of open-loop optimal controls. Manuscript submitted for publication. (2018).

[22] J. Sun and J. Yong, Linear quadratic stochastic differential games: open-loop and closed-loop saddle points. SIAM J. Control Optim. 52 (2014) 4082-4121.

[23] J. Sun and J. Yong, Stochastic Linear-Quadratic Optimal Control Theory: Open-Loop and Closed-Loop Solutions. Springer International Publishing (2020).

[24] S. Tang, General linear quadratic optimal stochastic control problems with random coefficients: Linear stochastic Hamilton systems and backward stochastic Riccati equations. SIAM J. Control Optim. 42 (2003) 53-75.

[25] S. Tang, Dynamic programming for general linear quadratic optimal stochastic control with random coefficients. SIAM J. Control Optim. 53 (2015) 1082-1106.

[26] T. Wang, Necessary conditions in stochastic linear quadratic problems and their applications. J. Math. Anal. Appl. 469 (2019) 280-297.

[27] W.M. Wonham, On a matrix riccati equation of stochastic control. SIAM J. Control 6 (1968) 681-697.

[28] Z. Wu and X.-R. Wang, FBSDE with Poisson process and its application to linear quadratic stochastic optimal control problem with random jumps. Acta Autom. Sin. 29 (2003) 821-826.

[29] G. Yin, X. Zhou, Markowitz's mean-variance portfolio selection with Regime switching: from discrete-time models to their continuous-time limits. IEEE Trans. Autom. Control. 49 (2004) 349-360.

[30] J. Yong and X. Zhou, Stochastic Controls: Hamiltonian Systems and HJB Equations. Springer, New York (1999).

[31] Z. Yu, Infinite horizon jump-diffusion forward-backward stochastic differential equations and their application to backward linear-quadratic problems. ESAIM: COCV 23 (2017) 1331-1359.

[32] C. Zalinescu, On uniformly convex functions. J. Math. Anal. Appl. 95 (1983) 344-374.

[33] C. Zalinescu, Convex Analysis in General Vector Spaces. World Scientific (2002). 
[34] Q. Zhang and G. Yin, On nearly optimal controls of hybrid LQG problems. IEEE Trans. Autom. Control. 44 (1999) $2271-2282$.

[35] X. Zhang, R.J. Elliott and T.K. Siu, A stochastic maximum principle for a Markov regime-switching jump-diffusion model and its application to finance. SIAM J. Control Optim. 50 (2012) 964-990.

[36] X. Zhang, R.J. Elliott, T.K. Siu and J. Guo, Markovian regime-switching market completion using additional Markov jump assets. IMA J. Manag. Math. 23 (2011) 283-305.

[37] X. Zhang, T.K. Siu and Q. Meng, Portfolio selection in the enlarged Markovian regime-switching market. SIAM J. Control Optim. 48 (2010) 3368-3388.

[38] X. Zhang, Z. Sun and J. Xiong, A general stochastic maximum principle for a Markov regime switching jump-diffusion model of mean-field type. SIAM J. Control Optim. 56 (2018) 2563-2592.

[39] X. Zhou and G. Yin, Markowitz's mean-variance portfolio selection with regime switching: a continuous-time model. SIAM J. Control Optim. 42 (2003) 1466-1482. 